\title{
APPROXIMATION OF THE PARETO OPTIMAL SET FOR MULTIOBJECTIVE OPTIMAL CONTROL PROBLEMS USING VIABILITY KERNELS
}

\author{
Alexis Guigue ${ }^{1}$
}

\begin{abstract}
This paper provides a convergent numerical approximation of the Pareto optimal set for finite-horizon multiobjective optimal control problems in which the objective space is not necessarily convex. Our approach is based on Viability Theory. We first introduce a set-valued return function $V$ and show that the epigraph of $V$ equals the viability kernel of a certain related augmented dynamical system. We then introduce an approximate set-valued return function with finite set-values as the solution of a multiobjective dynamic programming equation. The epigraph of this approximate set-valued return function equals to the finite discrete viability kernel resulting from the convergent numerical approximation of the viability kernel proposed in [P. Cardaliaguet, M. Quincampoix and P. Saint-Pierre. Birkhauser, Boston (1999) 177-247. P. Cardaliaguet, M. Quincampoix and P. Saint-Pierre, Set-Valued Analysis 8 (2000) 111-126]. As a result, the epigraph of the approximate set-valued return function converges to the epigraph of $V$. The approximate set-valued return function finally provides the proposed numerical approximation of the Pareto optimal set for every initial time and state. Several numerical examples illustrate our approach.
\end{abstract}

Mathematics Subject Classification. 49M2, 49L20, 54C60, 90C29.

Received March 9, 2012. Revised November 20, 2012.

Published online October 10, 2013.

\section{INTRODUCTION}

Many applications, such as trajectory planning for spacecraft [7] and robotic manipulators [15], continuous casting of steel [16], fishery management $[8,18]$, etc., involve optimal control formulations where $p$ objective functions $(p>1)$ need to be optimized simultaneously. For a General Optimization Problem (GOP) with a vector-valued objective function, the definition of an optimal solution requires the comparison between elements in the objective space, which is the set of all possible values that can be taken by the vector-valued objective function. This comparison is generally provided by a binary relation, expressing the preferences of the decision maker. In applications, it is common to consider the binary relation defined in terms of a pointed convex cone $P \subset \mathbf{R}^{p}$ containing the origin [23]. However, in this paper, for simplicity, we will only consider the case $P=\mathbf{R}_{+}^{p}$, which yields the well-known Pareto optimality. The resolution of (GOP) therefore consists of finding the set of Pareto optimal elements in the objective space, or Pareto optimal set. In general, this set cannot be

Keywords and phrases. Multiobjective optimal control, Pareto optimality, viability theory, convergent numerical approximation, dynamic programming.

1 Department of Mathematics, The University of British Columbia, Room 121, 1984 Mathematics Road, Vancouver, B.C.,

V6T 1Z2, Canada. aguigue@math.ubc.ca 
obtained analytically and we have to resort to numerical approximations. The main objective of this paper is therefore to propose a convergent numerical approximation of the Pareto optimal set for a general finite-horizon multiobjective optimal control problem (MOC). We take care to avoid making any convexity assumption on the objective space $Y$ (or more generally, on the set $Y+\mathbf{R}_{+}^{p}$ ). Indeed, in the case where the objective space (or $Y+\mathbf{R}_{+}^{p}$ ) is convex, simple methods such the weighting method can be used to generate the entire Pareto optimal set [20], Theorem 3.4.4.

When the objective space is not convex, very few approaches to find the Pareto optimal set have been proposed. An important line of research is to use evolutionary algorithms [9,11], such as genetic algorithms. Also, very recently, an approach [17] inspired by the $\epsilon$-constraint method in nonlinear multiobjective optimization [19], pp. 85-95 has been developed for multiobjective exit-time optimal control problems where $P=\mathbf{R}_{+}^{p}$. In this approach, the $n$-dimensional state is augmented by $p-1$ dimensions, which yields a new single objective optimal control problem. The Pareto optimal set of the original problem can be retrieved by inspecting the values of the return function of this new augmented problem. The return function of the new problem is finally approximated by solving numerically the corresponding $(n+p-1)$-dimensional Hamilton-Jacobi-Bellman equation using a semi-Lagrangian "marching" method.

In this paper, instead of an exit-time optimal control problem, we consider an optimal control problem over a fixed finite horizon $[0, T]$. The proposed approach differs fundamentally from the one in [17]. We use Viability Theory $[1,6]$ and the existing numerical schemes for approximating viability kernels $[4,5,10]$. Our first contribution is to reformulate the problem of finding the Pareto optimal set for (MOC) as the problem of determining a viability kernel. This is done by considering the epigraph of the return function $V(\cdot, \cdot)$ : $[0, T] \times \mathbf{R}^{n} \rightarrow 2^{\mathbf{R}^{p}}$, defined as the set-valued map associating with each time $t \in[0, T]$ and state $\mathbf{x} \in \mathbf{R}^{n}$ the set of Pareto optimal elements in the objective space $Y(t, \mathbf{x})$, where $Y(t, \mathbf{x})$ is the set of all possible values that can be taken by the vector-valued objective function for trajectories starting at $\mathbf{x}$ at time $t[13,14]$. Hence, the Pareto optimal set for any time $t$ and state $\mathbf{x}$ can be obtained just by evaluating $V$ at $(t, \mathbf{x})$. Our second contribution is to use the numerical schemes mentioned above to derive a convergent approximation of $V$. This approximation is a set-valued map with finite set-values, called the approximate set-valued return function. Hence, an approximation of the Pareto optimal set $V(t, \mathbf{x})$ can be obtained just by evaluating the approximate set-valued return function at $(t, \mathbf{x})$. The advantage of using Viability Theory is that it provides a framework that applies to problems with minimal regularity and convexity assumptions. Hence, it is expected that the proposed approach could be easily extended to more general classes of problems than the one considered in this paper, e.g., problems with state constraints, etc.

More precisely, the first step in the proposed approach is to show that the epigraph of $V$, i.e., the graph of the set-valued map $V+\mathbf{R}_{+}^{p}$, coincides with the viability kernel of a certain related augmented dynamical system. The next step is to introduce an approximate set-valued return function as the solution of a multiobjective dynamic programming equation. The epigraph of this function is shown to be equal to the finite discrete viability kernel resulting from the convergent numerical approximation of the viability kernel proposed in $[4,5]$. From there, we easily obtain that the epigraph of the approximate set-valued return function converges in the sense of Painlevé-Kuratowski to the epigraph of $V$. The multiobjective dynamic programming equation obtained is very similar to the one obtained in [12], where no proof of convergence was provided.

This paper is organized as follows. In Section 2, we briefly discuss the concept of optimality in multiobjective optimization and present several useful properties related to Pareto optimal sets. In Section 3, we detail the class of multiobjective optimal control problems considered and define $V$. In Section 4, we show that the epigraph of $V$ equals the viability kernel of a certain related augmented dynamical system. Following $[4,5]$, we then propose in Section 5 a finite discrete approximation of this viability kernel. In Section 6, we show that this approximation corresponds to the epigraph of a set-valued function that satisfies a certain multiobjective dynamic programming equation. From the latter equation, we derive in Section 7 a numerical algorithm to compute this set-valued function and therefore the approximate Pareto optimal set at $(t, \mathbf{x})$. Some numerical examples, for which the Pareto optimal set is analytically known, are provided in Section 8 and some conclusions are finally drawn in Section 9 . 


\section{Multiobjective Optimization}

For an optimization problem with a $p$-dimensional vector-valued objective function, the definition of an optimal solution requires a comparison between elements in the objective space, which is the set of all possible values that can be taken by the vector-valued objective function. This comparison is generally provided by a binary relation, expressing the preferences of the decision maker. In applications, it is common to define a binary preference relation in terms of a pointed convex cone $P \subset \mathbf{R}^{p}$ containing the origin [23].

Definition 2.1. Let $\mathbf{y}_{1}, \mathbf{y}_{\mathbf{2}} \in \mathbf{R}^{p}$. Then, $\mathbf{y}_{1} \preceq \mathbf{y}_{\mathbf{2}}$ if and only if $\mathbf{y}_{\mathbf{2}} \in \mathbf{y}_{\mathbf{1}}+P$.

The binary relation in Definition 2.1 yields the definition of generalized Pareto optimality.

Definition 2.2. Let $S$ be a nonempty subset of $\mathbf{R}^{p}$. An element $\mathbf{y}_{1} \in S$ is said to be a generalized Pareto optimal element of $S$ if and only if there is no $\mathbf{y}_{\mathbf{2}} \in S\left(\mathbf{y}_{\mathbf{2}} \neq \mathbf{y}_{\mathbf{1}}\right)$ such that $\mathbf{y}_{\mathbf{2}} \preceq \mathbf{y}_{\mathbf{1}}$, or equivalently, if and only if there is no $\mathbf{y}_{\mathbf{2}}$ such that $\mathbf{y}_{\mathbf{1}} \in \mathbf{y}_{\mathbf{2}}+P \backslash\{\mathbf{0}\}$. The set of generalized Pareto optimal elements of $S$ is called the generalized Pareto optimal set and is denoted by $\mathcal{E}(S, P)$. When $P=\mathbf{R}_{+}^{p}$, the generalized Pareto optimal elements are referred to simply as Pareto optimal elements, and the set $\mathcal{E}(S, P)$ is denoted $\mathcal{E}(S)$.

An important role in this paper is played by the external stability or domination property ([21], pp. 59-66).

Definition 2.3 (External stability). A nonempty subset $S$ of $\mathbf{R}^{p}$ is said to be externally stable if and only if

$$
S \subset \mathcal{E}(S, P)+P .
$$

An immediate consequence of the external stability property is that $S+P=\mathcal{E}(S, P)+P$. When $P$ is closed, a sufficient condition for a nonempty closed set $S$ to be externally stable is given in Proposition 2.5. Note that this condition is also sufficient to guarantee the existence of generalized Pareto optimal elements.

Definition 2.4 (Recession cone). Let $S$ be a nonempty subset of $\mathbf{R}^{p}$. The extended recession cone $S^{+}$is defined by

$$
S^{+}=\left\{\mathbf{y} \in \mathbf{R}^{p}: \exists h_{k} \rightarrow 0^{+}, \exists \mathbf{y}_{k} \in S, h_{k} \mathbf{y}_{k} \rightarrow \mathbf{y}\right\} .
$$

Proposition 2.5 ([21, Thm. 3.2.10]). Let $S$ be a nonempty closed subset of $\mathbf{R}^{p}$. If $P$ is closed and $S$ is $P$ bounded ([21], p. 52), i.e., $S^{+} \cap-P=\{\mathbf{0}\}$, then $S$ is externally stable.

Corollary 2.6. Let $K$ be a nonempty compact subset of $\mathbf{R}^{p}$. If $P$ is closed, then $K$ is externally stable.

In the case of finite sets, which are compact and therefore externally stable from Corollary 2.6, we have the two following results, which will be used to simplify the numerical algorithm proposed in Section 7 .

Lemma 2.7. Let $S_{1}$ and $S_{2}$ be two finite subsets of $\mathbf{R}^{p}$. Let $P$ be closed. Then, $\mathcal{E}\left(S_{1} \cup S_{2}, P\right)=\mathcal{E}\left(S_{1} \cup \mathcal{E}\left(S_{2}\right), P\right)$.

Proof. Take $\mathbf{z}_{\mathbf{1}} \in \mathcal{E}\left(S_{1} \cup S_{2}, P\right)$. Assume for contradiction that $\mathbf{z}_{\mathbf{1}} \notin \mathcal{E}\left(S_{1} \cup \mathcal{E}\left(S_{2}, P\right), P\right)$. Then, by external stability, there exists $\mathbf{z}_{\mathbf{2}} \in \mathcal{E}\left(S_{1} \cup \mathcal{E}\left(S_{2}, P\right), P\right) \subset S_{1} \cup S_{2}$ such that $\mathbf{z}_{\mathbf{1}} \in \mathbf{z}_{\mathbf{2}}+P \backslash\{\mathbf{0}\}$. But this contradicts $\mathbf{z}_{1} \in \mathcal{E}\left(S_{1} \cup S_{2}, P\right)$.

Conversely, take $\mathbf{z}_{\mathbf{1}} \in \mathcal{E}\left(S_{1} \cup \mathcal{E}\left(S_{2}\right), P\right)$. Assume for contradiction that $\mathbf{z}_{\mathbf{1}} \notin \mathcal{E}\left(S_{1} \cup S_{2}, P\right)$. Then, by external stability, there exists $\mathbf{z}_{\mathbf{2}} \in \mathcal{E}\left(S_{1} \cup S_{2}, P\right) \subset S_{1} \cup S_{2}$ such that $\mathbf{z}_{\mathbf{1}} \in \mathbf{z}_{\mathbf{2}}+P \backslash\{\mathbf{0}\}$. Assume that $\mathbf{z}_{\mathbf{2}} \notin S_{2}$. Then, necessarily $\mathbf{z}_{\mathbf{2}} \in S_{1} \cup \mathcal{E}\left(S_{2}, P\right)$. But, this contradicts $\mathbf{z}_{\mathbf{1}} \in \mathcal{E}\left(S_{1} \cup \mathcal{E}\left(S_{2}, P\right), P\right)$. Assume now that $\mathbf{z}_{\mathbf{2}} \in S_{2}$. Then, by external stability, there exists $\mathbf{z}_{\mathbf{3}} \in \mathcal{E}\left(S_{2}, P\right)$ such that $\mathbf{z}_{\mathbf{2}} \in \mathbf{z}_{\mathbf{3}}+P \backslash\{\mathbf{0}\}$. Hence, $\mathbf{z}_{\mathbf{1}} \in \mathbf{z}_{\mathbf{3}}+P \backslash\{\mathbf{0}\}$ with $\mathbf{z}_{3} \in S_{1} \cup \mathcal{E}\left(S_{2}, P\right)$. But this again contradicts $\mathbf{z}_{\mathbf{1}} \in \mathcal{E}\left(S_{1} \cup \mathcal{E}\left(S_{2}, P\right), P\right)$. 
Proposition 2.8. Let $S_{1}, \ldots, S_{I}$ be finite subsets of $\mathbf{R}^{p}$. Let $P$ be closed. Then,

$$
\mathcal{E}\left(\bigcup_{i=1}^{I} S_{i}, P\right)=E_{I},
$$

where $E_{I}$ is recursively defined by $E_{1}=\mathcal{E}\left(S_{1}, P\right)$ and the relation

$$
E_{i+1}=\mathcal{E}\left(S_{i+1} \cup E_{i}, P\right) .
$$

Proof. We proceed by induction. For $I=1$, this is by definition. Assume now that the relation holds up to $I$. We aim to prove that it holds for $I+1$, i.e.,

$$
\mathcal{E}\left(\bigcup_{i=1}^{I+1} S_{i}\right)=E_{I+1}
$$

Apply Lemma 2.7 to $\bigcup_{i=1}^{I} S_{i}$ and $S_{I+1}$. Then, we get

$$
\mathcal{E}\left(\bigcup_{i=1}^{I+1} S_{i}, P\right)=\mathcal{E}\left(S_{I+1} \cup \mathcal{E}\left(\bigcup_{i=1}^{I} S_{i}, P\right), P\right) .
$$

Using the induction assumption, this yields

$$
\mathcal{E}\left(\bigcup_{i=1}^{I+1} S_{i}, P\right)=\mathcal{E}\left(S_{I+1} \cup E_{I}, P\right)=E_{I+1} .
$$

In this paper, for simplicity, we will only consider the case $P=\mathbf{R}_{+}^{p}$.

\section{A multiobjective finite-horizon optimal CONTROL PROBLEM}

In this paper, we will take for $\|\cdot\|$ in $\mathbf{R}^{p}$ and $\mathbf{R}^{n}$ the supremum norm. Let $\mathbf{B}$ be the closed unit ball.

Consider the evolution over a fixed finite time interval $I=[0, T](0<T<\infty)$ of an autonomous dynamical system [3] whose $n$-dimensional state dynamics are given by a continuous function $\mathbf{f}(\cdot, \cdot): \mathbf{R}^{n} \times U \rightarrow \mathbf{R}^{n}$, where the control space $U$ is a nonempty compact subset of $\mathbf{R}^{m}$. The function $\mathbf{f}(\cdot, \mathbf{u})$ is assumed to be Lipschitz, i.e., some $K_{\mathbf{f}}>0$ obeys

$$
\forall \mathbf{u} \in U, \forall \mathbf{x}_{1}, \mathbf{x}_{\mathbf{2}} \in \mathbf{R}^{n},\left\|\mathbf{f}\left(\mathbf{x}_{1}, \mathbf{u}\right)-\mathbf{f}\left(\mathbf{x}_{\mathbf{2}}, \mathbf{u}\right)\right\| \leq K_{\mathbf{f}}\left\|\mathbf{x}_{\mathbf{1}}-\mathbf{x}_{\mathbf{2}}\right\| .
$$

We also assume that the function $\mathbf{f}$ is uniformly bounded, i.e., some $M_{\mathbf{f}}>0$ obeys

$$
\forall \mathbf{x} \in \mathbf{R}^{n}, \forall \mathbf{u} \in U,\|\mathbf{f}(\mathbf{x}, \mathbf{u})\| \leq M_{\mathbf{f}}
$$

A control $\mathbf{u}(\cdot): I \rightarrow U$ is a Lebesgue measurable function. The set of controls is denoted by $\mathcal{U}$. The continuity of $\mathbf{f}$ and the Lipschitz condition (3.1) guarantee that, given any $t \in I$, initial state $\mathbf{x} \in \mathbf{R}^{n}$, and control $\mathbf{u}(\cdot) \in \mathcal{U}$, the initial value problem,

$$
\left\{\begin{array}{l}
\dot{\mathbf{x}}(s)=\mathbf{f}(\mathbf{x}(s), \mathbf{u}(s)) \text { a.e. } s \in[t, T] \\
\mathbf{x}(t)=\mathbf{x}
\end{array}\right.
$$

has a unique solution, called a trajectory, which we denote $s \rightarrow \mathbf{x}(s ; t, \mathbf{x}, \mathbf{u}(\cdot))$.

The cost of a trajectory over $[t, T], t \in I$, is given by a vector-valued function $\mathbf{J}(\cdot, \cdot, \cdot): I \times \mathbf{R}^{n} \times \mathcal{U} \rightarrow \mathbf{R}^{p}$,

$$
\mathbf{J}(t, \mathbf{x}, \mathbf{u}(\cdot))=\int_{t}^{T} \mathbf{L}(\mathbf{x}(s ; \mathbf{x}, \mathbf{u}(\cdot)), \mathbf{u}(s)) \mathrm{d} s,
$$


where the vector-valued function $\mathbf{L}(\cdot, \cdot): \mathbf{R}^{n} \times U \rightarrow \mathbf{R}^{p}$, called the running cost, is assumed to be continuous. For simplicity, no terminal cost is included in (3.4). We assume that the function $\mathbf{L}$ is uniformly bounded, i.e., some $M_{\mathbf{L}} \geq 0$ obeys

$$
\forall \mathbf{x} \in \mathbf{R}^{n}, \forall \mathbf{u} \in U,\|\mathbf{L}(\mathbf{x}, \mathbf{u})\| \leq M_{\mathbf{L}},
$$

and that the function $\mathbf{L}(\cdot, \mathbf{u})$ satisfies a Lipschitz condition, i.e., some $K_{\mathbf{L}} \geq 0$ obeys

$$
\forall \mathbf{u} \in U, \forall \mathbf{x}_{1}, \mathbf{x}_{2} \in \mathbf{R}^{n},\left\|\mathbf{L}\left(\mathbf{x}_{1}, \mathbf{u}\right)-\mathbf{L}\left(\mathbf{x}_{2}, \mathbf{u}\right)\right\| \leq K_{\mathbf{L}}\left\|\mathbf{x}_{1}-\mathbf{x}_{\mathbf{2}}\right\| .
$$

The objective space $Y(t, \mathbf{x})$ for (MOC) is defined as the set of all possible costs for all possible controls:

$$
Y(t, \mathbf{x})=\{\mathbf{J}(t, \mathbf{x}, \mathbf{u}(\cdot)), \mathbf{u}(\cdot) \in \mathcal{U}\}
$$

From (3.5), it follows that the set $Y(t, \mathbf{x})$ is bounded (by $M_{\mathbf{L}} T$ ), and also that $Y(t, \mathbf{x}) \subset\left\{-(T-t) M_{\mathbf{L}} \mathbf{1}\right\}+\mathbf{R}_{+}^{p}$. However, the set $Y(t, \mathbf{x})$ is not necessarily closed.

The set-valued return function $V(\cdot, \cdot): I \times \mathbf{R}^{n} \rightarrow 2^{\mathbf{R}^{p}}$ for (MOC) is defined as the set-valued map which associates with each time $t \in I$ and initial state $\mathbf{x} \in \mathbf{R}^{n}$ the set of Pareto optimal elements in the objective space $Y(t, \mathbf{x})$ :

$$
V(t, \mathbf{x})=\mathcal{E}(\operatorname{cl}(Y(t, \mathbf{x}))) .
$$

The closure in (3.7) is used to guarantee the existence of Pareto optimal elements [14], Proposition 3.5. Hence, $\forall t \in I, \forall \mathbf{x} \in \mathbf{R}^{n}, V(t, \mathbf{x}) \neq \emptyset$.

Remark 3.1. When $p=1$, (3.7) takes the form

$$
V(t, \mathbf{x})=\left\{\inf _{\mathbf{u}(\cdot) \in \mathcal{U}} \int_{t}^{T} L(\mathbf{x}(s ; t, \mathbf{x}, \mathbf{u}(\cdot)), \mathbf{u}(s)) \mathrm{d} s\right\} .
$$

Hence, $V(t, \mathbf{x})=\{v(t, \mathbf{x})\}$, where $v(\cdot, \cdot)$ is the value function for single objective optimal control problems [3,22].

Finally, as $V(t, \mathbf{x}) \subset \operatorname{cl}(Y(t, \mathbf{x}))$, we have

$$
V(t, \mathbf{x}) \subset\left\{-(T-t) M_{\mathbf{L}} \mathbf{1}\right\}+\mathbf{R}_{+}^{p} .
$$

The objective of this paper is to find a convergent approximation to the Pareto optimal set $V\left(0, \mathbf{x}_{\mathbf{0}}\right)$ where $\mathbf{x}_{\mathbf{0}} \in \mathbf{R}^{n}$ is some given initial state.

\section{Characterization of the Set-Valued Return FUnCtion}

In this section, we show that the epigraph of the set-valued return function $V$, i.e., the graph of the set-valued map $V+\mathbf{R}_{+}^{p}$, is equal to the viability kernel of a certain related augmented dynamical system.

Define two set-valued maps $\mathrm{FL}^{-}$and $\mathrm{FL}^{+}$from $\mathbf{R}^{n}$ to $\mathbf{R}^{n} \times \mathbf{R}^{p}$ by

$$
\mathrm{FL}^{-}(\mathbf{x})=\overline{\mathrm{co}}\left(\bigcup_{\mathbf{u} \in U}\{(\mathbf{f}(\mathbf{x}, \mathbf{u}),-\mathbf{L}(\mathbf{x}, \mathbf{u}))\}\right),
$$

and

$$
\mathrm{FL}^{+}(\mathbf{x})=\overline{c 0}\left(\bigcup_{\mathbf{u} \in U}\{(\mathbf{f}(\mathbf{x}, \mathbf{u}), \mathbf{L}(\mathbf{x}, \mathbf{u}))\}\right),
$$

where $\overline{\mathrm{co}}(S)$ denotes the closure of the convex hull of the set $S$. Observe that these two set-valued maps take convex compact nonempty values. Moreover, they are bounded by $M_{\mathrm{FL}}=\max \left\{M_{\mathbf{f}}, M_{\mathbf{L}}\right\}$ and Lipschitz 
with Lipschitz constant $K_{\mathrm{FL}}=\max \left\{K_{\mathbf{f}}, K_{\mathbf{L}}\right\}$. Define also an expanded set-valued map from $\mathbf{R} \times \mathbf{R}^{n} \times \mathbf{R}^{p}$ to $\mathbf{R} \times \mathbf{R}^{n} \times \mathbf{R}^{p}$ by

$$
\phi(t, \mathbf{x}, \mathbf{z})= \begin{cases}\{1\} \times \mathrm{FL}^{-}(\mathbf{x}) & \text { if } t<T, \\ {[0,1] \times \overline{\mathrm{co}}\left(\mathrm{FL}^{-}(\mathbf{x}) \cup\{(\mathbf{0}, \mathbf{0})\}\right)} & \text { if } t \geq T .\end{cases}
$$

It is easy to see that $\phi$ is a Marchaud map ([4], Def. 2.2) bounded by $\max \left\{1, M_{\mathrm{FL}}\right\}$.

Consider now the differential inclusion

$$
(\dot{t}(s), \dot{\mathbf{x}}(s), \dot{\mathbf{z}}(s)) \in \phi(t(s), \mathbf{x}(s), \mathbf{z}(s)) \text { a.e. } s \geq 0,
$$

and the closed set $\mathcal{H}$ by

$$
\mathcal{H}=\left\{(t, \mathbf{x}, \mathbf{z}): t \in[0, T], \mathbf{x} \in \mathbf{R}^{n}, \mathbf{z} \in\left\{-(T-t) M_{\mathbf{L}} \mathbf{1}\right\}+\mathbf{R}_{+}^{p}\right\} .
$$

Proposition 4.1. The epigraph of the set-valued map $V$ equals the viability kernel of $\mathcal{H}$ for $\phi$, i.e.,

$$
\operatorname{Epi}(V)=\operatorname{Viab}_{\phi}(\mathcal{H}),
$$

where $\operatorname{Epi}(V)$, by definition, $\operatorname{Epi}(V)=\operatorname{Graph}\left(V+\mathbf{R}_{+}^{p}\right)$.

Proof. First, we prove the inclusion

$$
\operatorname{Viab}_{\phi}(\mathcal{H}) \subset \operatorname{Graph}\left(V+\mathbf{R}_{+}^{p}\right) .
$$

Take $(t, \mathbf{x}, \mathbf{z}) \in \operatorname{Viab}_{\phi}(\mathcal{H})$. Of course $(t, \mathbf{x}, \mathbf{z}) \in \mathcal{H}$, so $t \in I$ and $\mathbf{z} \in\left\{-(T-t) M_{\mathbf{L}} \mathbf{1}\right\}+\mathbf{R}_{+}^{p}$.

- Assume that $t=T$. Then, as $V(T, \mathbf{x})=\{\mathbf{0}\}$, it follows that $\mathbf{z} \in \mathbf{R}_{+}^{p}=V(T, \mathbf{x})+\mathbf{R}_{+}^{p}$.

- Assume that $t \in[0, T)$. Let $(t(\cdot), \mathbf{x}(\cdot), \mathbf{z}(\cdot))$ be a solution to $(4.2)$ with initial condition $(t, \mathbf{x}, \mathbf{z})$ which remains in $\mathcal{H}$. By definition of $\phi,(\mathbf{x}(\cdot), \mathbf{z}(\cdot))$ is a solution to the differential inclusion

$$
\begin{cases}(\dot{\mathbf{x}}(s), \dot{\mathbf{z}}(s)) & \in \mathrm{FL}^{-}(\mathbf{x}(s)) \text { a.e. } s \in[0, T-t], \\ \mathbf{x}(0) & =\mathbf{x}, \\ \mathbf{z}(0) & =\mathbf{z},\end{cases}
$$

while $t(s)=s+t$. Let $s^{\prime}=s+t$. For $s^{\prime} \in[t, T]$, define $\mathbf{x}^{\prime}\left(s^{\prime}\right)=\mathbf{x}\left(s^{\prime}-t\right)$ and $\mathbf{z}^{\prime}\left(s^{\prime}\right)=\mathbf{z}\left(s^{\prime}-t\right)$. Then, $\left(\mathbf{x}^{\prime}(\cdot), \mathbf{z}^{\prime}(\cdot)\right)$ satisfies the differential inclusion

$$
\left\{\begin{aligned}
\left(\dot{\mathbf{x}}^{\prime}\left(s^{\prime}\right), \dot{\mathbf{z}}^{\prime}\left(s^{\prime}\right)\right) & \in \mathrm{FL}^{-}\left(\mathbf{x}^{\prime}\left(s^{\prime}\right)\right) \text { a.e. } s^{\prime} \in[t, T] \\
\mathbf{x}^{\prime}(t) & =\mathbf{x} \\
\mathbf{z}^{\prime}(t) & =\mathbf{z}
\end{aligned}\right.
$$

By the Relaxation Theorem [22], Theorem 2.7.2, for each fixed $\epsilon>0$, there exists $\mathbf{u}(\cdot) \in \mathcal{U}$ such that

$$
\left\|\mathbf{x}^{\prime}(\cdot)-\mathbf{x}(\cdot ; t, \mathbf{x}, \mathbf{u}(\cdot))\right\| \leq \epsilon \text { and }\left\|\mathbf{z}^{\prime}(\cdot)-\mathbf{z}(\cdot ; t,(\mathbf{x}, \mathbf{z}), \mathbf{u}(\cdot))\right\| \leq \epsilon
$$

(the initial state for the evolving vector $(\mathbf{x}(\cdot), \mathbf{z}(\cdot))$ is $(\mathbf{x}, \mathbf{z})$, but we have simplified the notation by noting only the relevant components for each block).

In particular, we get

$$
\mathbf{z}^{\prime}(T) \leq \mathbf{z}(T ; t,(\mathbf{x}, \mathbf{z}), \mathbf{u}(\cdot))+\epsilon \mathbf{1} .
$$

Moreover, as $\left(s^{\prime}, \mathbf{x}^{\prime}\left(s^{\prime}\right), \mathbf{z}^{\prime}\left(s^{\prime}\right)\right) \in \mathcal{H}$ for all $s^{\prime} \in[t, T]$, we have

$$
\mathbf{z}^{\prime}\left(s^{\prime}\right) \in\left\{-\left(T-s^{\prime}\right) M_{\mathbf{L}} \mathbf{1}\right\}+\mathbf{R}_{+}^{p},
$$

or

$$
\mathbf{z}^{\prime}(T) \in \mathbf{R}_{+}^{p} .
$$


Hence,

or

$$
\mathbf{z}(T ; t,(\mathbf{x}, \mathbf{z}), \mathbf{u}(\cdot))=\mathbf{z}-\int_{t}^{T} \mathbf{L}\left(\mathbf{x}\left(s^{\prime} ; t, \mathbf{x}, \mathbf{u}(\cdot)\right), \mathbf{u}\left(s^{\prime}\right)\right) \mathrm{d} s^{\prime} \geq \mathbf{z}^{\prime}(T)-\epsilon \mathbf{1} \geq-\epsilon \mathbf{1},
$$

$$
\mathbf{z}+\epsilon \mathbf{1} \geq \int_{t}^{T} \mathbf{L}\left(\mathbf{x}\left(s^{\prime} ; t, \mathbf{x}, \mathbf{u}(\cdot)\right), \mathbf{u}\left(s^{\prime}\right)\right) \mathrm{d} s^{\prime} .
$$

Therefore $\mathbf{z}+\epsilon \mathbf{1} \in Y(t, \mathbf{x})+\mathbf{R}_{+}^{p}$ for each $\epsilon>0$. This implies that $\mathbf{z} \in \operatorname{cl}(Y(t, \mathbf{x}))+\mathbf{R}_{+}^{p}=V(t, \mathbf{x})+\mathbf{R}_{+}^{p}$ by external stability.

Second, we prove the inclusion

$$
\operatorname{Graph}\left(V+\mathbf{R}_{+}^{p}\right) \subset \operatorname{Viab}_{\phi}(\mathcal{H}) .
$$

Take $(t, \mathbf{x}, \mathbf{z}) \in \operatorname{Graph}\left(V+\mathbf{R}_{+}^{p}\right)$. Hence, $t \in[0, T]$ and from (3.8), $\mathbf{z} \in\left\{-(T-t) M_{\mathbf{L}} \mathbf{1}\right\}+\mathbf{R}_{+}^{p}$.

- Assume that $t=T$. Then $\mathbf{z} \in \mathbf{R}_{+}^{p}$, so $(t(\cdot), \mathbf{x}(\cdot), \mathbf{z}(\cdot))=(T, \mathbf{x}, \mathbf{z})$ is a solution to (4.2) viable in $\mathcal{H}$.

- Assume that $t \in[0, T)$. We have $\mathbf{z}=\mathbf{z}^{\prime}+\mathbf{d}$, where $\mathbf{z}^{\prime} \in V(t, \mathbf{x})$ and $\mathbf{d} \in \mathbf{R}_{+}^{p}$. By definition of $V(t, \mathbf{x})$, there exists a sequence $\mathbf{u}_{n}(\cdot) \in \mathcal{U}$ such that

$$
\lim _{n \rightarrow+\infty} \int_{t}^{T} \mathbf{L}\left(\mathbf{x}_{n}\left(s ; t, \mathbf{x}, \mathbf{u}_{n}(\cdot)\right), \mathbf{u}_{n}(s)\right) \mathrm{d} s=\mathbf{z}^{\prime} .
$$

Using the Compactness of Trajectories Theorem [22], Theorem 2.5.3 and by passing to a subsequence if necessary, we can assume that there exists $(\mathbf{x}(\cdot), \mathbf{z}(\cdot))$ solution on $[t, T]$ to the differential inclusion

$$
\left(\dot{\mathbf{x}}\left(s^{\prime}\right), \dot{\mathbf{z}}\left(s^{\prime}\right)\right) \in \mathrm{FL}^{+}\left(\mathbf{x}\left(s^{\prime}\right)\right) \text { a.e. } s^{\prime} \in[t, T]
$$

with initial condition $(\mathbf{x}, \mathbf{0})$ such that

$$
\lim _{n \rightarrow+\infty}\left\|\mathbf{x}_{n}\left(\cdot ; t, \mathbf{x}, \mathbf{u}_{n}(\cdot)\right)-\mathbf{x}(\cdot)\right\|=0
$$

and

$$
\lim _{n \rightarrow+\infty}\left\|\mathbf{z}_{n}\left(\cdot ; t,(\mathbf{x}, \mathbf{0}), \mathbf{u}_{n}(\cdot)\right)-\mathbf{z}(\cdot)\right\|=0 .
$$

From (4.3) and (4.4), we deduce that $\mathbf{z}(T)=\mathbf{z}^{\prime}$.

Define now $\left(\mathbf{x}^{\prime}(\cdot), \mathbf{z}^{\prime}(\cdot)\right)$ as follows:

$$
\left(t(s), \mathbf{x}^{\prime}(s), \mathbf{z}^{\prime}(s)\right)= \begin{cases}(s+t, \mathbf{x}(s+t), \mathbf{z}-\mathbf{z}(s+t)) & s \in[0, T-t], \\ (T, \mathbf{x}(T), \mathbf{d}) & s>T-t .\end{cases}
$$

As $\mathbf{z}^{\prime}(T-t)=\mathbf{z}-\mathbf{z}(T)=\mathbf{z}-\mathbf{z}^{\prime}=\mathbf{d}$, it follows that $\left(t(\cdot), \mathbf{x}^{\prime}(\cdot), \mathbf{z}^{\prime}(\cdot)\right)$ is a solution of (4.2). It remains to check that $\left(t(s), \mathbf{x}^{\prime}(s), \mathbf{z}^{\prime}(s)\right) \in \mathcal{H}$ for all $s \geq 0$. For $s>T-t$, as $\mathbf{d} \in \mathbf{R}_{+}^{p}$, this is obvious. For $s \in[0, T-t]$, using the definition of $\mathbf{z},(4.3)$, and (4.4), we have

$$
\mathbf{z}^{\prime}(s)=\lim _{n \rightarrow+\infty} \int_{t}^{T} \mathbf{L}\left(\mathbf{x}_{n}\left(s ; t, \mathbf{x}, \mathbf{u}_{n}(\cdot)\right), \mathbf{u}_{n}(s)\right) \mathrm{d} s+\mathbf{d}-\mathbf{z}_{n}\left(s+t ; t,(\mathbf{x}, \mathbf{0}), \mathbf{u}_{n}(\cdot)\right) .
$$

As

we get

$$
\mathbf{z}_{n}\left(s+t ; t,(\mathbf{x}, \mathbf{0}), \mathbf{u}_{n}(\cdot)\right)=\int_{t}^{s+t} \mathbf{L}\left(\mathbf{x}_{n}\left(s ; t, \mathbf{x}, \mathbf{u}_{n}(\cdot)\right), \mathbf{u}_{n}(s)\right) \mathrm{d} s
$$

$$
\mathbf{z}^{\prime}(s)=\mathbf{d}+\lim _{n \rightarrow+\infty} \int_{s+t}^{T} \mathbf{L}\left(\mathbf{x}_{n}\left(s ; t, \mathbf{x}, \mathbf{u}_{n}(\cdot)\right), \mathbf{u}_{n}(s)\right) \mathrm{d} s .
$$


As

$$
\int_{s+t}^{T} \mathbf{L}\left(\mathbf{x}_{n}\left(s ; t, \mathbf{x}, \mathbf{u}_{n}(\cdot)\right), \mathbf{u}_{n}(s)\right) \mathrm{d} s \geq-(T-(s+t)) M_{\mathbf{L}} \mathbf{1}
$$

and $\mathbf{d} \in \mathbf{R}_{+}^{p}$, we finally obtain $\mathbf{z}^{\prime}(s) \geq-(T-t(s)) M_{\mathbf{L}} \mathbf{1}$. Hence, $\left(t(s), \mathbf{x}^{\prime}(s), \mathbf{z}^{\prime}(s)\right) \in \mathcal{H}$ and $\left(t(\cdot), \mathbf{x}^{\prime}(\cdot), \mathbf{z}^{\prime}(\cdot)\right)$ is viable in $\mathcal{H}$.

Remark 4.2. Proposition 4.1 remains valid if we take for $\mathcal{H}$ the closed set $\left\{(t, \mathbf{x}, \mathbf{z}): t \in[0, T], \mathbf{x} \in \mathbf{R}^{n}, \mathbf{z} \in\right.$ $\left.\left\{-(T-t) \bar{M}_{\mathbf{L}} \mathbf{1}\right\}+\mathbf{R}_{+}^{p}\right\}$, where $\bar{M}_{\mathbf{L}}>M_{\mathbf{L}}$. This remark will be used in Section 6 .

\section{Approximation of $\operatorname{Viab}_{\phi}(\mathcal{H})$}

In this section, we approximate $\operatorname{Viab}_{\phi}(\mathcal{H})$ by finite discrete viability kernels. This is done two stages. First we replace the original differential inclusion system (4.2) by a finite difference inclusion system defined by a certain set-valued map $G_{\epsilon}$. Then we associate with $G_{\epsilon}$ a suitable finite set-valued map $\Gamma_{\epsilon, h}$ defined on finite sets. The finite discrete viability kernel for this set-valued map approximates $\operatorname{Viab}_{\phi}(\mathcal{H})$.

\subsection{Approximation of $\operatorname{Viab}_{\phi}(\mathcal{H})$ by discrete viability kernels}

Theorem 2.14 in [4] shows how $\operatorname{Viab}_{\phi}(\mathcal{H})$ can be approximated by discrete viability kernels in the sense of Painlevé-Kuratowski by considering an approximation $\phi_{\epsilon}$ of $\phi$ satisfying the following three properties:

$\left(\mathbf{H}_{\mathbf{0}}\right) \quad \phi_{\epsilon}$ is an upper semicontinuous set-valued map from $\mathbf{R} \times \mathbf{R}^{n} \times \mathbf{R}^{p}$ to $\mathbf{R} \times \mathbf{R}^{n} \times \mathbf{R}^{p}$ whose values are nonempty convex compact sets.

$$
\operatorname{Graph}\left(\phi_{\epsilon}\right) \subset \operatorname{Graph}(\phi)+g(\epsilon) \mathbf{B} \text { and } \lim _{\epsilon \rightarrow 0^{+}} g(\epsilon)=0^{+} .
$$

$\left(\mathbf{H}_{2}\right)$

$$
\forall\left(t_{\epsilon}, \mathbf{x}_{\epsilon}, \mathbf{z}_{\epsilon}\right) \in \mathbf{R} \times \mathbf{R}^{n} \times \mathbf{R}^{p}, \bigcup_{\left\|(t, \mathbf{x}, \mathbf{z})-\left(t_{\epsilon}, \mathbf{x}_{\epsilon}, \mathbf{z}_{\epsilon}\right)\right\| \leq M \epsilon} \phi(t, \mathbf{x}, \mathbf{z}) \subset \phi_{\epsilon}\left(t_{\epsilon}, \mathbf{x}_{\epsilon}, \mathbf{z}_{\epsilon}\right),
$$

where $M=\max \left\{1, M_{\mathrm{FL}}\right\}$ denotes a bound for $\phi$ and $\epsilon>0$ is the time step discretization.

We now demonstrate that these conditions hold for the set-valued map $\phi_{\epsilon}, \epsilon>0$, from $\mathbf{R} \times \mathbf{R}^{n} \times \mathbf{R}^{p}$ to $\mathbf{R} \times \mathbf{R}^{n} \times \mathbf{R}^{p}$ defined by

$$
\phi_{\epsilon}(t, \mathbf{x}, \mathbf{z})= \begin{cases}\{1\} \times\left(\mathrm{FL}^{-}(\mathbf{x})+\epsilon K M \mathbf{B}\right), & \text { if } t<T-M \epsilon, \\ {[0,1] \times \overline{\mathrm{co}}\left(\left(\mathrm{FL}^{-}(\mathbf{x})+\epsilon K M \mathbf{B}\right) \cup\{(\mathbf{0}, \mathbf{0})\}\right),} & \text { if } t \geq T-M \epsilon .\end{cases}
$$

Theorem 5.1. The set-valued map $\phi_{\epsilon}$ satisfies $\left(\mathbf{H}_{\mathbf{0}}\right),\left(\mathbf{H}_{\mathbf{1}}\right)$, and $\left(\mathbf{H}_{\mathbf{2}}\right)$.

Proof.

$\left(\mathbf{H}_{\mathbf{0}}\right)$ This follows from the properties of the set-valued map $\mathrm{FL}^{-}$and the fact that $\mathrm{FL}^{-}(\mathbf{x})+\epsilon K M \mathbf{B} \subset$ $\overline{\mathrm{CO}}\left(\left(\mathrm{FL}^{-}(\mathbf{x})+\epsilon K M \mathbf{B}\right) \cup\{(\mathbf{0}, \mathbf{0})\}\right)$.

$\left(\mathbf{H}_{\mathbf{1}}\right)$ This relation holds with $g(\epsilon)=\epsilon \max \{1, K\} M$. To see this, let $\left(t_{\epsilon}, \mathbf{x}_{\epsilon}, \mathbf{z}_{\epsilon}\right) \in \mathbf{R} \times \mathbf{R}^{n} \times \mathbf{R}^{p}$ and $\left(s_{\epsilon}, \mathbf{f}_{\epsilon}, \mathbf{l}_{\epsilon}\right) \in$ $\phi_{\epsilon}\left(t_{\epsilon}, \mathbf{x}_{\epsilon}, \mathbf{z}_{\epsilon}\right)$. Two cases arise:

- $t_{\epsilon}<T-M \epsilon$. Here $s_{\epsilon}=1$ and $\left(\mathbf{f}_{\epsilon}, \mathbf{l}_{\epsilon}\right) \in \mathrm{FL}^{-}\left(\mathbf{x}_{\epsilon}\right)+\epsilon K M \mathbf{B}$, so $g(\epsilon)=\epsilon K M$.

- $t_{\epsilon} \geq T-M \epsilon$. Here we have $s_{\epsilon} \in[0,1]$ and $\left(\mathbf{f}_{\epsilon}, \mathbf{l}_{\epsilon}\right) \in \overline{\mathrm{Co}}\left(\left(\mathrm{FL}^{-}\left(\mathbf{x}_{\epsilon}\right)+\epsilon K M \mathbf{B}\right) \cup\{(\mathbf{0}, \mathbf{0})\}\right)$. There exists $t \geq T$ such that $\left|t-t_{\epsilon}\right| \leq M \epsilon$. Thus

$$
\begin{aligned}
\left(\mathbf{f}_{\epsilon}, \mathbf{l}_{\epsilon}\right) & \in \overline{\mathrm{co}}\left(\left(\mathrm{FL}^{-}\left(\mathbf{x}_{\epsilon}\right)+\epsilon K M \mathbf{B}\right) \cup\{(\mathbf{0}, \mathbf{0})\}\right), \\
& \subset \overline{\mathrm{co}}\left(\mathrm{FL}^{-}\left(\mathbf{x}_{\epsilon}\right) \cup\{(\mathbf{0}, \mathbf{0})\}\right)+\epsilon K M \mathbf{B} .
\end{aligned}
$$

The choice $g(\epsilon)=\epsilon \max \{1, K\} M$ covers both alternatives. 
$\left(\mathbf{H}_{\mathbf{2}}\right)$ Let $\left(t_{\epsilon}, \mathbf{x}_{\epsilon}, \mathbf{z}_{\epsilon}\right) \in \mathbf{R} \times \mathbf{R}^{n} \times \mathbf{R}^{p}$. Take $(t, \mathbf{x}, \mathbf{z}) \in \mathbf{R} \times \mathbf{R}^{n} \times \mathbf{R}^{p}$ such that $\left\|(t, \mathbf{x}, \mathbf{z})-\left(t_{\epsilon}, \mathbf{x}_{\epsilon}, \mathbf{z}_{\epsilon}\right)\right\| \leq M \epsilon$. In particular, $\left\|\mathbf{x}-\mathbf{x}_{\epsilon}\right\| \leq M \epsilon$. Two cases arise:

- $t_{\epsilon}<T-M \epsilon$. This implies $t<T$. The Lipschitz property of $\mathrm{FL}^{-}$yields:

$$
\phi(t, \mathbf{x}, \mathbf{z})=\{1\} \times \mathrm{FL}^{-}(\mathbf{x}) \subset\{1\} \times\left(\mathrm{FL}^{-}\left(\mathbf{x}_{\epsilon}\right)+\epsilon K M \mathbf{B}\right)=\phi_{\epsilon}\left(t_{\epsilon}, \mathbf{x}_{\epsilon}, \mathbf{z}_{\epsilon}\right) .
$$

- $t_{\epsilon} \geq T-M \epsilon$. If, in addition, $t<T$, then as above,

$$
\phi(t, \mathbf{x}, \mathbf{z})=\{1\} \times \mathrm{FL}^{-}(\mathbf{x}) \subset\{1\} \times\left(\mathrm{FL}^{-}\left(\mathbf{x}_{\epsilon}\right)+\epsilon K M \mathbf{B}\right) \subset \phi_{\epsilon}\left(t_{\epsilon}, \mathbf{x}_{\epsilon}, \mathbf{z}_{\epsilon}\right) .
$$

Alternatively, suppose $t \geq T$. Then, using the Lipschitz property of $\mathrm{FL}^{-}$, we have

$$
\mathrm{FL}^{-}(\mathbf{x}) \cup\{(\mathbf{0}, \mathbf{0})\} \subset\left(\mathrm{FL}^{-}\left(\mathbf{x}_{\epsilon}\right)+\epsilon K M \mathbf{B}\right) \cup\{(\mathbf{0}, \mathbf{0})\} .
$$

Hence,

$$
\phi(t, \mathbf{x}, \mathbf{z})=[0,1] \times \overline{\mathrm{co}}\left(\mathrm{FL}^{-}(\mathbf{x}) \cup\{(\mathbf{0}, \mathbf{0})\}\right) \subset \phi_{\epsilon}\left(t_{\epsilon}, \mathbf{x}_{\epsilon}, \mathbf{z}_{\epsilon}\right) .
$$

\subsection{Approximation of $\operatorname{Viab}_{\phi}(\mathcal{H})$ by discrete finite viability kernels}

Theorem 2.19 in [4] goes on to show how $\operatorname{Viab}_{\phi}(\mathcal{H})$ can be approximated by finite discrete viability kernels in the sense of Painlevé-Kuratowski by considering an approximation $\Gamma_{\epsilon, h}$ of $G_{\epsilon}(\cdot)$ satisfying the following two properties:

$\left(\mathbf{H}_{3}\right)$

$$
\operatorname{Graph}\left(\Gamma_{\epsilon, h}\right) \subset \operatorname{Graph}\left(G_{\epsilon}\right)+\psi(\epsilon, h) \mathbf{B} \text { and } \lim _{\epsilon \rightarrow 0^{+}, \frac{h}{\epsilon} \rightarrow 0^{+}} \frac{\psi(\epsilon, h)}{\epsilon}=0^{+} .
$$

$\left(\mathbf{H}_{\mathbf{4}}\right) \forall\left(t_{h}, \mathbf{x}_{\mathbf{h}}, \mathbf{z}_{\mathbf{h}}\right) \in \mathbf{R}_{h} \times \mathbf{R}_{h}^{n} \times \mathbf{R}_{h}^{p}$,

$$
\bigcup_{\left\|\left(t_{\epsilon}, \mathbf{x}_{\epsilon}, \mathbf{z}_{\epsilon}\right)-\left(t_{h}, \mathbf{x}_{\mathbf{h}}, \mathbf{z}_{\mathbf{h}}\right)\right\| \leq h}\left(G_{\epsilon}\left(t_{\epsilon}, \mathbf{x}_{\epsilon}, \mathbf{z}_{\epsilon}\right)+h \mathbf{B}\right) \cap \mathbf{R}_{h} \times \mathbf{R}_{h}^{n} \times \mathbf{R}_{h}^{p} \subset \Gamma_{\epsilon, h}\left(t_{h}, \mathbf{x}_{\mathbf{h}}, \mathbf{z}_{\mathbf{h}}\right),
$$

where $h>0$ is the state step discretization, $\mathbf{R}_{h}=\{k h, k \in \mathbf{Z}\}$, and $G_{\epsilon}$ is the set-valued map from $\mathbf{R} \times \mathbf{R}^{n} \times \mathbf{R}^{p}$ to $\mathbf{R} \times \mathbf{R}^{n} \times \mathbf{R}^{p}$ defined by

$$
G_{\epsilon}\left(t_{\epsilon}, \mathbf{x}_{\epsilon}, \mathbf{z}_{\epsilon}\right)=\left\{\left(t_{\epsilon}, \mathbf{x}_{\epsilon}, \mathbf{z}_{\epsilon}\right)\right\}+\epsilon \phi_{\epsilon}\left(t_{\epsilon}, \mathbf{x}_{\epsilon}, \mathbf{z}_{\epsilon}\right) .
$$

We apply this result to the set-valued map $\Gamma_{\epsilon, h}$ from $\mathbf{R}_{h} \times \mathbf{R}_{h}^{n} \times \mathbf{R}_{h}^{p}$ to $\mathbf{R}_{h} \times \mathbf{R}_{h}^{n} \times \mathbf{R}_{h}^{p}$ defined as follows:

- If $t_{h}<T-M \epsilon-h, \Gamma_{\epsilon, h}\left(t_{h}, \mathbf{x}_{\mathbf{h}}, \mathbf{z}_{\mathbf{h}}\right)=$

$$
\left[t_{h}+\epsilon-2 h, t_{h}+\epsilon+2 h\right] \cap \mathbf{R}_{h} \times\left(\left\{\left(\mathbf{x}_{\mathbf{h}}, \mathbf{z}_{\mathbf{h}}\right)\right\}+\epsilon \mathrm{FL}^{-}\left(\mathbf{x}_{\mathbf{h}}\right)+\alpha_{\epsilon, h} \mathbf{B}\right) \cap \mathbf{R}_{h}^{n} \times \mathbf{R}_{h}^{p} .
$$

- If $t_{h} \geq T-M \epsilon-h, \Gamma_{\epsilon, h}\left(t_{h}, \mathbf{x}_{\mathbf{h}}, \mathbf{z}_{\mathbf{h}}\right)=$

$$
\left[t_{h}, t_{h}+\epsilon+2 h\right] \cap \mathbf{R}_{h} \times \overline{\mathrm{co}}\left(\left(\left\{\left(\mathbf{x}_{\mathbf{h}}, \mathbf{z}_{\mathbf{h}}\right)\right\}+\epsilon \mathrm{FL}^{-}\left(\mathbf{x}_{\mathbf{h}}\right)+\alpha_{\epsilon, h} \mathbf{B}\right) \cup\left(\left\{\left(\mathbf{x}_{\mathbf{h}}, \mathbf{z}_{\mathbf{h}}\right)\right\}+2 h \mathbf{B}\right)\right) \cap \mathbf{R}_{h}^{n} \times \mathbf{R}_{h}^{p},
$$

where $\alpha_{\epsilon, h}=2 h+\epsilon h K+\epsilon^{2} K M$.

We assume that $\epsilon>2 h$.

Theorem 5.2. The set-valued map $\Gamma_{\epsilon, h}$ satisfies $\left(\mathbf{H}_{\mathbf{3}}\right)$ and $\left(\mathbf{H}_{\mathbf{4}}\right)$.

Proof. 
$\left(\mathbf{H}_{3}\right)$ This relation holds with $\psi(\epsilon, h)=4 h+\epsilon h K$, which verifies

$$
\lim _{\epsilon \rightarrow 0^{+}, \frac{h}{\epsilon} \rightarrow 0^{+}} \frac{(4+\epsilon K) h}{\epsilon}=0^{+} .
$$

Let $\left(t_{h}, \mathbf{x}_{\mathbf{h}}, \mathbf{z}_{\mathbf{h}}\right) \in \mathbf{R}_{h} \times \mathbf{R}_{h}^{n} \times \mathbf{R}_{h}^{p}$ and $\left(s_{h}, \mathbf{f}_{\mathbf{h}}, \mathbf{l}_{\mathbf{h}}\right) \in \Gamma_{\epsilon, h}\left(t_{h}, \mathbf{x}_{\mathbf{h}}, \mathbf{z}_{\mathbf{h}}\right)$. Two cases arise:

- $t_{h}<T-M \epsilon-h$. Then, $s_{h} \in\left[t_{h}+\epsilon-2 h, t_{h}+\epsilon+2 h\right] \cap \mathbf{R}_{h} \subset t_{h}+\epsilon+[-2 h, 2 h]$, and

$$
\begin{aligned}
\left(\mathbf{f}_{\mathbf{h}}, \mathbf{l}_{\mathbf{h}}\right) & \in\left(\left\{\left(\mathbf{x}_{\mathbf{h}}, \mathbf{z}_{\mathbf{h}}\right)\right\}+\epsilon \mathrm{FL}^{-}\left(\mathbf{x}_{\mathbf{h}}\right)+\alpha_{\epsilon, h} \mathbf{B}\right) \cap \mathbf{R}_{h}^{n} \times \mathbf{R}_{h}^{p}, \\
& \subset\left\{\left(\mathbf{x}_{\mathbf{h}}, \mathbf{z}_{\mathbf{h}}\right)\right\}+\epsilon \mathrm{FL}^{-}\left(\mathbf{x}_{\mathbf{h}}\right)+\alpha_{\epsilon, h} \mathbf{B}, \\
& =\left\{\left(\mathbf{x}_{\mathbf{h}}, \mathbf{z}_{\mathbf{h}}\right)\right\}+\epsilon\left(\mathrm{FL}^{-}\left(\mathbf{x}_{\mathbf{h}}\right)+\epsilon K M \mathbf{B}\right)+(2 h+\epsilon h K) \mathbf{B} .
\end{aligned}
$$

Hence, $\left(s_{h}, \mathbf{f}_{\mathbf{h}}, \mathbf{l}_{\mathbf{h}}\right) \in\left(t_{h}, \mathbf{x}_{\mathbf{h}}, \mathbf{z}_{\mathbf{h}}\right)+\epsilon \phi_{\epsilon}\left(t_{h}, \mathbf{x}_{\mathbf{h}}, \mathbf{z}_{\mathbf{h}}\right)+\max \{2 h, 2 h+\epsilon h K\} \mathbf{B}=G_{\epsilon}\left(t_{h}, \mathbf{x}_{\mathbf{h}}, \mathbf{z}_{\mathbf{h}}\right)+(2 h+\epsilon h K) \mathbf{B}$. - $t_{h} \geq T-M \epsilon-h$. We have $s_{h} \in\left[t_{h}, t_{h}+\epsilon+2 h\right] \cap \mathbf{R}_{h}$ and

$$
\left(\mathbf{f}_{\mathbf{h}}, \mathbf{l}_{\mathbf{h}}\right) \in \overline{\operatorname{co}}\left(\left(\left\{\left(\mathbf{x}_{\mathbf{h}}, \mathbf{z}_{\mathbf{h}}\right)\right\}+\epsilon \mathrm{FL}^{-}\left(\mathbf{x}_{\mathbf{h}}\right)+\alpha_{\epsilon, h} \mathbf{B}\right) \cup\left(\left\{\left(\mathbf{x}_{\mathbf{h}}, \mathbf{z}_{\mathbf{h}}\right)\right\}+h \mathbf{B}\right)\right) \cap \mathbf{R}_{h}^{n} \times \mathbf{R}_{h}^{p} .
$$

There exists $t_{\epsilon} \geq T-M \epsilon$ such that $0 \leq t_{\epsilon}-t_{h} \leq h$. We have

$$
\begin{aligned}
s_{h} & \in\left[t_{h}, t_{h}+\epsilon+2 h\right] \cap \mathbf{R}_{h}, \\
& \subset\left[t_{h}, t_{h}+\epsilon+2 h\right], \\
& \subset t_{\epsilon}+\epsilon[0,1]+[-2 h, 2 h] .
\end{aligned}
$$

Moreover,

$$
\begin{aligned}
\left(\mathbf{f}_{\mathbf{h}}, \mathbf{l}_{\mathbf{h}}\right) & \in \overline{\mathrm{co}}\left(\left(\left\{\left(\mathbf{x}_{\mathbf{h}}, \mathbf{z}_{\mathbf{h}}\right)\right\}+\epsilon \mathrm{FL}^{-}\left(\mathbf{x}_{\mathbf{h}}\right)+\alpha_{\epsilon, h} \mathbf{B}\right) \cup\left(\left\{\left(\mathbf{x}_{\mathbf{h}}, \mathbf{z}_{\mathbf{h}}\right)\right\}+2 h \mathbf{B}\right)\right) \cap \mathbf{R}_{h}^{n} \times \mathbf{R}_{h}^{p}, \\
& \subset \overline{\mathrm{co}}\left(\left(\left\{\left(\mathbf{x}_{\mathbf{h}}, \mathbf{z}_{\mathbf{h}}\right)\right\}+\epsilon \mathrm{FL}^{-}\left(\mathbf{x}_{\mathbf{h}}\right)+\alpha_{\epsilon, h} \mathbf{B}\right) \cup\left(\left\{\left(\mathbf{x}_{\mathbf{h}}, \mathbf{z}_{\mathbf{h}}\right)\right\}+2 h \mathbf{B}\right)\right), \\
& =\left\{\left(\mathbf{x}_{\mathbf{h}}, \mathbf{z}_{\mathbf{h}}\right)\right\}+\overline{\mathrm{co}}\left(\left(\epsilon \mathrm{FL}^{-}\left(\mathbf{x}_{\mathbf{h}}\right)+\alpha_{\epsilon, h} \mathbf{B}\right) \cup(\{(\mathbf{0}, \mathbf{0})\}+2 h \mathbf{B})\right), \\
& =\left\{\left(\mathbf{x}_{\mathbf{h}}, \mathbf{z}_{\mathbf{h}}\right)\right\}+\overline{\mathrm{co}}\left(\left(\epsilon\left(\mathrm{FL}^{-}\left(\mathbf{x}_{\mathbf{h}}\right)+\epsilon K M \mathbf{B}\right)+(2 h+\epsilon h K) \mathbf{B}\right) \cup(\{(\mathbf{0}, \mathbf{0})\}+2 h \mathbf{B})\right), \\
& \subset\left\{\left(\mathbf{x}_{\mathbf{h}}, \mathbf{z}_{\mathbf{h}}\right)\right\}+\overline{\mathrm{co}}\left(\left(\epsilon\left(\mathrm{FL}^{-}\left(\mathbf{x}_{\mathbf{h}}\right)+\epsilon K M \mathbf{B}\right)\right) \cup\{(\mathbf{0}, \mathbf{0})\}\right)+(4 h+\epsilon h K) \mathbf{B} .
\end{aligned}
$$

Hence, $\left(s_{h}, \mathbf{f}_{\mathbf{h}}, \mathbf{l}_{\mathbf{h}}\right) \in\left(t_{\epsilon}, \mathbf{x}_{\mathbf{h}}, \mathbf{z}_{\mathbf{h}}\right)+\epsilon \phi_{\epsilon}\left(t_{\epsilon}, \mathbf{x}_{\mathbf{h}}, \mathbf{z}_{\mathbf{h}}\right)+\max \{4 h+\epsilon h K, 2 h\} \mathbf{B}=G_{\epsilon}\left(t_{\epsilon}, \mathbf{x}_{\mathbf{h}}, \mathbf{z}_{\mathbf{h}}\right)+(4 h+\epsilon h K) \mathbf{B}$.

$\left(\mathbf{H}_{\mathbf{4}}\right)$ Let $\left(t_{h}, \mathbf{x}_{\mathbf{h}}, \mathbf{z}_{\mathbf{h}}\right) \in \mathbf{R}_{h} \times \mathbf{R}_{h}^{n} \times \mathbf{R}_{h}^{p}$. Take $\left(t_{\epsilon}, \mathbf{x}_{\epsilon}, \mathbf{z}_{\epsilon}\right) \in \mathbf{R} \times \mathbf{R}^{n} \times \mathbf{R}^{p}$ such that $\left\|\left(t_{\epsilon}, \mathbf{x}_{\epsilon}, \mathbf{z}_{\epsilon}\right)-\left(t_{h}, \mathbf{x}_{\mathbf{h}}, \mathbf{z}_{\mathbf{h}}\right)\right\| \leq h$. In particular, $\left|t_{\epsilon}-t_{h}\right| \leq h$ and $\left\|\mathbf{x}-\mathbf{x}_{\mathbf{h}}\right\| \leq h$. Two cases arise:

- $t_{h}<T-M \epsilon-h$. This implies $t_{\epsilon}<T-M \epsilon$. The Lipschitz property of $\mathrm{FL}^{-}$yields:

$$
\begin{aligned}
G_{\epsilon}\left(t_{\epsilon}, \mathbf{x}_{\epsilon}, \mathbf{z}_{\epsilon}\right)+h \mathbf{B} & =\left(t_{\epsilon}, \mathbf{x}_{\epsilon}, \mathbf{z}_{\epsilon}\right)+\epsilon \phi_{\epsilon}\left(t_{\epsilon}, \mathbf{x}_{\epsilon}, \mathbf{z}_{\epsilon}\right)+h \mathbf{B}, \\
& =\left(t_{\epsilon}+\epsilon+[-h, h]\right) \times\left(\left\{\left(\mathbf{x}_{\epsilon}, \mathbf{z}_{\epsilon}\right)\right\}+\epsilon \mathrm{FL}^{-}\left(\mathbf{x}_{\epsilon}\right)+\epsilon^{2} K M \mathbf{B}+h \mathbf{B}\right), \\
& \subset\left(t_{h}+\epsilon+[-2 h, 2 h]\right) \times\left(\left\{\left(\mathbf{x}_{h}, \mathbf{z}_{h}\right)\right\}+h \mathbf{B}+\epsilon \mathrm{FL}^{-}\left(\mathbf{x}_{h}\right)+\epsilon h K \mathbf{B}+\epsilon^{2} K M+h \mathbf{B}\right), \\
& =\left(t_{h}+\epsilon+[-2 h, 2 h]\right) \times\left(\left\{\left(\mathbf{x}_{h}, \mathbf{z}_{h}\right)\right\}+\epsilon \mathrm{FL}^{-}\left(\mathbf{x}_{h}\right)+\alpha_{\epsilon, h} \mathbf{B}\right) .
\end{aligned}
$$

Hence,

$$
\left(G_{\epsilon}\left(t_{\epsilon}, \mathbf{x}_{\epsilon}, \mathbf{z}_{\epsilon}\right)+h \mathbf{B}\right) \cap \mathbf{R}_{h} \times \mathbf{R}_{h}^{n} \times \mathbf{R}_{h}^{p} \subset \Gamma_{\epsilon, h}\left(t_{h}, \mathbf{x}_{\mathbf{h}}, \mathbf{z}_{\mathbf{h}}\right) .
$$

- $t_{h} \geq T-M \epsilon-h$. If, in addition, $t_{\epsilon}<T-M \epsilon$, then as above,

$$
G_{\epsilon}\left(t_{\epsilon}, \mathbf{x}_{\epsilon}, \mathbf{z}_{\epsilon}\right)+h \mathbf{B} \subset\left(t_{h}+\epsilon+[-2 h, 2 h]\right) \times\left(\left\{\left(\mathbf{x}_{h}, \mathbf{z}_{h}\right)\right\}+\epsilon \mathrm{FL}^{-}\left(\mathbf{x}_{h}\right)+\alpha_{\epsilon, h} \mathbf{B}\right) .
$$


As $\epsilon>2 h$,

$$
t_{h}+\epsilon+[-2 h, 2 h] \subset\left[t_{h}, t_{h}+\epsilon+2 h\right] .
$$

Moreover,

$$
\left\{\left(\mathbf{x}_{h}, \mathbf{z}_{h}\right)\right\}+\epsilon \mathrm{FL}^{-}\left(\mathbf{x}_{h}\right)+\alpha_{\epsilon, h} \mathbf{B} \subset \overline{\mathrm{co}}\left(\left(\left\{\left(\mathbf{x}_{\mathbf{h}}, \mathbf{z}_{\mathbf{h}}\right)\right\}+\epsilon \mathrm{FL}^{-}\left(\mathbf{x}_{\mathbf{h}}\right)+\alpha_{\epsilon, h} \mathbf{B}\right) \cup\left(\left\{\left(\mathbf{x}_{\mathbf{h}}, \mathbf{z}_{\mathbf{h}}\right)\right\}+2 h \mathbf{B}\right)\right) .
$$

Hence,

$$
\left(G_{\epsilon}\left(t_{\epsilon}, \mathbf{x}_{\epsilon}, \mathbf{z}_{\epsilon}\right)+h \mathbf{B}\right) \cap \mathbf{R}_{h} \times \mathbf{R}_{h}^{n} \times \mathbf{R}_{h}^{p} \subset \Gamma_{\epsilon, h}\left(t_{h}, \mathbf{x}_{\mathbf{h}}, \mathbf{z}_{\mathbf{h}}\right) .
$$

Alternatively, $t_{\epsilon} \geq T-M \epsilon$. In this case, using the Lipschitz property of $\mathrm{FL}^{-}$, we have

$$
\begin{aligned}
G_{\epsilon}\left(t_{\epsilon}, \mathbf{x}_{\epsilon}, \mathbf{z}_{\epsilon}\right)+h \mathbf{B} & =\left(t_{\epsilon}, \mathbf{x}_{\epsilon}, \mathbf{z}_{\epsilon}\right)+\epsilon\left([0,1] \times \overline{\mathrm{co}}\left(\left(\mathrm{FL}^{-}\left(\mathbf{x}_{\epsilon}\right)+\epsilon K M \mathbf{B}\right) \cup\{(\mathbf{0}, \mathbf{0})\}\right)\right)+h \mathbf{B}, \\
& =\left(\left[t_{\epsilon}-h, t_{\epsilon}+\epsilon+h\right]\right) \times\left(\overline{\mathrm{co}}\left(\left\{\left(\mathbf{x}_{\epsilon}, \mathbf{z}_{\epsilon}\right)+\epsilon \mathrm{FL}^{-}\left(\mathbf{x}_{\epsilon}\right)+\epsilon^{2} K M \mathbf{B}\right\} \cup\left\{\left(\mathbf{x}_{\epsilon}, \mathbf{z}_{\epsilon}\right)\right\}\right)+h \mathbf{B}\right) .
\end{aligned}
$$

Now,

$$
\begin{aligned}
& \overline{\mathrm{co}}\left(\left\{\left(\mathbf{x}_{\epsilon}, \mathbf{z}_{\epsilon}\right)+\epsilon \mathrm{FL}^{-}\left(\mathbf{x}_{\epsilon}\right)+\epsilon^{2} K M \mathbf{B}\right\} \cup\left\{\left(\mathbf{x}_{\epsilon}, \mathbf{z}_{\epsilon}\right)\right\}\right)+h \mathbf{B}, \\
& \subset \overline{\mathrm{co}}\left(\left\{\left(\mathbf{x}_{h}, \mathbf{z}_{h}\right)+h \mathbf{B}+\epsilon \mathrm{FL}^{-}\left(\mathbf{x}_{h}\right)+\epsilon K h+\epsilon^{2} K M \mathbf{B}\right\} \cup\left(\left\{\left(\mathbf{x}_{h}, \mathbf{z}_{h}\right)\right\}+h \mathbf{B}\right)\right)+h \mathbf{B}, \\
& \subset \overline{\mathrm{co}}\left(\left\{\left(\mathbf{x}_{h}, \mathbf{z}_{h}\right)+\epsilon \mathrm{FL}^{-}\left(\mathbf{x}_{h}\right)+\alpha_{\epsilon, h} \mathbf{B}\right\} \cup\left(\left\{\left(\mathbf{x}_{h}, \mathbf{z}_{h}\right)\right\}+2 h \mathbf{B}\right)\right) .
\end{aligned}
$$

Hence,

$$
\left(G_{\epsilon}\left(t_{\epsilon}, \mathbf{x}_{\epsilon}, \mathbf{z}_{\epsilon}\right)+h \mathbf{B}\right) \cap \mathbf{R}_{h} \times \mathbf{R}_{h}^{n} \times \mathbf{R}_{h}^{p} \subset \Gamma_{\epsilon, h}\left(t_{h}, \mathbf{x}_{\mathbf{h}}, \mathbf{z}_{\mathbf{h}}\right) .
$$

\section{Convergent approximation of the Set-valued Return Function $V$}

In this section, we first introduce a sequence of approximate set-valued return functions with finite set-values recursively defined by a multiobjective dynamic programming equation $[13,14]$. We then show that the epigraphs of these approximate set-valued return functions are equal to the sets involved in the calculation of the finite discrete viability kernels of the discrete set $\mathcal{H}_{h}=(\mathcal{H}+h \mathbf{B}) \cap \mathbf{R}_{h} \times \mathbf{R}_{h}^{n} \times \mathbf{R}_{h}^{p}$ for the finite discrete dynamics $\Gamma_{\epsilon, h}$ ([4], Prop. 2.18). This allows us to conclude that the sequence of approximate set-valued return functions is finite and that the epigraph of the final approximate set-valued return function of this sequence converges to the epigraph of the set-valued return function $V$ in the sense of Painlevé-Kuratowski.

Recall the definition of $\mathcal{H}=\left\{(t, \mathbf{x}, \mathbf{z}): t \in[0, T], \mathbf{x} \in \mathbf{R}^{n}, \mathbf{z} \in\left\{-(T-t) M_{\mathbf{L}} \mathbf{1}\right\}+\mathbf{R}_{+}^{p}\right\}$. Here, we take $\bar{M}_{\mathbf{L}}>$ $M_{\mathbf{L}}$ (Rem. 4.2) in the definition of $\mathcal{H}$. Hence, $\mathcal{H}=\left\{(t, \mathbf{x}, \mathbf{z}): t \in[0, T], \mathbf{x} \in \mathbf{R}^{n}, \mathbf{z} \in\left\{-(T-t) \bar{M}_{\mathbf{L}} \mathbf{1}\right\}+\mathbf{R}_{+}^{p}\right\}$. Let $I_{h}=(I+[-h, h]) \cap \mathbf{R}_{h}$. We define the finite-valued set-valued map $V_{\epsilon, h}^{0}$ from $I_{h} \times \mathbf{R}_{h}^{n}$ to $\mathbf{R}_{h}^{p}$ such that

$$
\operatorname{Graph}\left(V_{\epsilon, h}^{0}+\mathbf{R}_{h,+}^{p}\right)=\mathcal{H}_{h}
$$

where $\mathcal{H}_{h}=(\mathcal{H}+h \mathbf{B}) \cap \mathbf{R}_{h} \times \mathbf{R}_{h}^{n} \times \mathbf{R}_{h}^{p}$. We now recursively define the finite set-valued maps $V_{\epsilon, h}^{k}, k \geq 1$, from $I_{h} \times \mathbf{R}_{h}^{n}$ to $\mathbf{R}_{h}^{p}$ as follows:

- If $t_{h}<T-M \epsilon-h, V_{\epsilon, h}^{k+1}\left(t_{h}, \mathbf{x}_{\mathbf{h}}\right)=$

$$
\mathcal{E}\left(\left\{\left(\epsilon \mathbf{l}+\alpha_{\epsilon, h} \mathbf{B}\right) \cap \mathbf{R}_{h}^{p}+V_{\epsilon, h}^{k}\left(\left(t_{h}+\epsilon+[-2 h, 2 h]\right) \cap \mathbf{R}_{h},\left(\mathbf{x}_{\mathbf{h}}+\epsilon \mathbf{f}+\alpha_{\epsilon, h} \mathbf{B}\right) \cap \mathbf{R}_{h}^{n}\right):(\mathbf{f}, \mathbf{l}) \in \mathrm{FL}^{+}\left(\mathbf{x}_{\mathbf{h}}\right)\right\}\right) .
$$

- Otherwise,

$$
V_{\epsilon, h}^{k+1}\left(t_{h}, \mathbf{x}_{\mathbf{h}}\right)=V_{\epsilon, h}^{k}\left(t_{h}, \mathbf{x}_{\mathbf{h}}\right)
$$


Remark 6.1. The closure is not required in (6.1) as the sets involved are finite.

We aim in the following two propositions to prove that

$$
\operatorname{Graph}\left(V_{\epsilon, h}^{k+1}+\mathbf{R}_{h,+}^{p}\right) \subset \operatorname{Graph}\left(V_{\epsilon, h}^{k}+\mathbf{R}_{h,+}^{p}\right),
$$

and

$$
\operatorname{Graph}\left(V_{\epsilon, h}^{k}+\mathbf{R}_{h,+}^{p}\right)=A^{k},
$$

where the sets $A^{k}$ are recursively defined ([4], Prop. 2.18) from $A^{0}=\mathcal{H}_{h}$ and the relation

$$
A^{k+1}=\left\{\left(t_{h}, \mathbf{x}_{\mathbf{h}}, \mathbf{z}_{\mathbf{h}}\right) \in A^{k}: \Gamma_{\epsilon, h}\left(t_{h}, \mathbf{x}_{\mathbf{h}}, \mathbf{z}_{\mathbf{h}}\right) \cap A^{k} \neq \emptyset\right\} .
$$

To simplify the proof of Proposition 6.2, we will assume that $T$ is a multiple of $h$ and that $\epsilon-2 h>2 h$, which guarantees that $\forall t_{h} \in I_{h}, t_{h}+\epsilon-2 h>h$. We will also assume that for all $\left(t_{h}, \mathbf{x}_{\mathbf{h}}\right) \in I_{h} \times \mathbf{R}_{h}^{n}$ such that $t_{h} \geq h$,

$$
V_{\epsilon, h}^{0}\left(t_{h}, \mathbf{x}_{\mathbf{h}}\right)=\left\{\left(-\left(T+h-t_{h}\right) \bar{M}_{\mathbf{L}}-h\right) \mathbf{1}\right\} .
$$

This guarantees that, for all $\left(t_{h}, \mathbf{x}_{\mathbf{h}}\right) \in I_{h} \times \mathbf{R}_{h}^{n}$, for all $(\mathbf{f}, \mathbf{l}) \in \mathrm{FL}^{+}\left(\mathbf{x}_{\mathbf{h}}\right)$, for all $\widetilde{t}_{h} \in\left(t_{h}+\epsilon+[-2 h, 2 h]\right) \cap \mathbf{R}_{h}$, for all $\widetilde{\mathbf{x}}_{\mathbf{h}} \in\left(\mathbf{x}_{\mathbf{h}}+\epsilon \mathbf{f}+\alpha_{\epsilon, h}\right) \cap \mathbf{R}_{h}^{n}$,

$$
V_{\epsilon, h}^{0}\left(\widetilde{t}_{h}, \widetilde{\mathbf{x}}_{\mathbf{h}}\right)=\left\{\left(-\left(T+h-\widetilde{t}_{h}\right) \bar{M}_{\mathbf{L}}-h\right) \mathbf{1}\right\} .
$$

We will finally assume that $\epsilon, h$, and $\bar{M}_{\mathbf{L}}$ have been chosen such that

$$
\epsilon M_{\mathbf{L}}+\alpha_{\epsilon, h} \leq(\epsilon-2 h) \bar{M}_{\mathbf{L}} .
$$

Proposition 6.2.

$$
\forall k, \operatorname{Graph}\left(V_{\epsilon, h}^{k+1}+\mathbf{R}_{h,+}^{p}\right) \subset \operatorname{Graph}\left(V_{\epsilon, h}^{k}+\mathbf{R}_{h,+}^{p}\right) .
$$

Proof. We start by proving that this relation holds for $k=0$. Take $\left(t_{h}, \mathbf{x}_{\mathbf{h}}, \mathbf{z}_{\mathbf{h}}\right) \in \operatorname{Graph}\left(V_{\epsilon, h}^{1}+\mathbf{R}_{h,+}^{p}\right)$. Hence, $\mathbf{z}_{\mathbf{h}} \in V_{\epsilon, h}^{1}\left(t_{h}, \mathbf{x}_{\mathbf{h}}\right)+\mathbf{R}_{h,+}^{p}$. Two cases arise:

1. If $t_{h} \geq T-M \epsilon-h$. From (6.2), we directly get $\mathbf{z}_{\mathbf{h}} \in V_{\epsilon, h}^{0}\left(t_{h}, \mathbf{x}_{\mathbf{h}}\right)+\mathbf{R}_{h,+}^{p}$.

2. Otherwise, $t_{h}<T-M \epsilon-h$. By (6.1),

$$
V_{\epsilon, h}^{1}\left(t_{h}, \mathbf{x}_{\mathbf{h}}\right)=\mathcal{E}\left(\left\{\left(\epsilon \mathbf{l}+\alpha_{\epsilon, h} \mathbf{B}\right) \cap \mathbf{R}_{h}^{p}+V_{\epsilon, h}^{0}\left(\left(t_{h}+\epsilon+[-2 h, 2 h]\right) \cap \mathbf{R}_{h},\left(\mathbf{x}_{\mathbf{h}}+\epsilon \mathbf{f}+\alpha_{\epsilon, h} \mathbf{B}\right) \cap \mathbf{R}_{h}^{n}\right):(\mathbf{f}, \mathbf{l}) \in \mathrm{FL}^{+}\left(\mathbf{x}_{\mathbf{h}}\right)\right\}\right),
$$

Let $\widetilde{t}_{h} \in\left(t_{h}+\epsilon+[-2 h, 2 h]\right) \cap \mathbf{R}_{h}, \widetilde{\mathbf{x}}_{\mathbf{h}} \in\left(\mathbf{x}_{\mathbf{h}}+\epsilon \mathbf{f}+\alpha_{\epsilon, h} \mathbf{B}\right) \cap \mathbf{R}_{h}^{n}, \overline{\mathbf{z}}_{\mathbf{h}} \in V_{\epsilon, h}^{0}\left(\widetilde{t}_{h}, \widetilde{\mathbf{x}}_{\mathbf{h}}\right)$, and $\widetilde{\mathbf{z}}_{\mathbf{h}} \in\left(\epsilon \mathbf{l}+\alpha_{\epsilon, h} \mathbf{B}\right) \cap \mathbf{R}_{h}^{p}$. Hence, $\widetilde{\mathbf{z}}_{\mathbf{h}} \geq-\left(\epsilon M_{\mathbf{L}}+\alpha_{\epsilon, h}\right) \mathbf{1}$. Moreover, from above, we have

$\overline{\mathbf{z}}_{\mathbf{h}}=\left(-\left(T+h-\widetilde{t}_{h}\right) \bar{M}_{\mathbf{L}}-h\right) \mathbf{1} \geq\left(-\left(T+h-t_{h}-(\epsilon-2 h)\right) \bar{M}_{\mathbf{L}}-h\right) \mathbf{1}=\left(-\left(T+h-t_{h}\right) \bar{M}_{\mathbf{L}}-h\right) \mathbf{1}+(\epsilon-2 h) \bar{M}_{\mathbf{L}} \mathbf{1}$.

Hence, by (6.3),

$\overline{\mathbf{z}}_{\mathbf{h}} \geq\left(-\left(T+h-t_{h}\right) \bar{M}_{\mathbf{L}}-h\right) \mathbf{1}+(\epsilon-2 h) \bar{M}_{\mathbf{L}} \mathbf{1}-\left(\epsilon M_{\mathbf{L}}+\alpha_{\epsilon, h}\right) \mathbf{1}+\left(\epsilon M_{\mathbf{L}}+\alpha_{\epsilon, h}\right) \mathbf{1} \geq-\left(T+h-t_{h}\right) \bar{M}_{\mathbf{L}} \mathbf{1}+\left(\epsilon M_{\mathbf{L}}+\alpha_{\epsilon, h}\right) \mathbf{1}$.

Therefore,

$$
\widetilde{\mathbf{z}}_{\mathbf{h}}+\overline{\mathbf{z}}_{\mathbf{h}} \in V_{\epsilon, h}^{0}\left(t_{h}, \mathbf{x}_{\mathbf{h}}\right)+\mathbf{R}_{h,+}^{p} .
$$

Hence,

$$
V_{\epsilon, h}^{1}\left(t_{h}, \mathbf{x}_{\mathbf{h}}\right) \subset V_{\epsilon, h}^{0}\left(t_{h}, \mathbf{x}_{\mathbf{h}}\right)+\mathbf{R}_{h,+}^{p},
$$

from which we get that

$$
\mathbf{z}_{\mathbf{h}} \in V_{\epsilon, h}^{1}\left(t_{h}, \mathbf{x}_{\mathbf{h}}\right)+\mathbf{R}_{h,+}^{p} \subset V_{\epsilon, h}^{0}\left(t_{h}, \mathbf{x}_{\mathbf{h}}\right)+\mathbf{R}_{h,+}^{p}+\mathbf{R}_{h,+}^{p}=V_{\epsilon, h}^{0}\left(t_{h}, \mathbf{x}_{\mathbf{h}}\right)+\mathbf{R}_{h,+}^{p} .
$$


Assume now that the desired relation holds up to $k$. We aim to prove that it holds for $k+1$, i.e.,

$$
\operatorname{Graph}\left(V_{\epsilon, h}^{k+2}+\mathbf{R}_{h,+}^{p}\right) \subset \operatorname{Graph}\left(V_{\epsilon, h}^{k+1}+\mathbf{R}_{h,+}^{p}\right) .
$$

Take $\left(t_{h}, \mathbf{x}_{\mathbf{h}}, \mathbf{z}_{\mathbf{h}}\right) \in \operatorname{Graph}\left(V_{\epsilon, h}^{k+2}+\mathbf{R}_{h,+}^{p}\right)$. Hence, $\mathbf{z}_{\mathbf{h}} \in V_{\epsilon, h}^{k+2}\left(t_{h}, \mathbf{x}_{\mathbf{h}}\right)+\mathbf{R}_{h,+}^{p}$. Two cases arise:

1. If $t_{h} \geq T-M \epsilon-h$. From (6.2), we directly get $\mathbf{z}_{\mathbf{h}} \in V_{\epsilon, h}^{k+1}\left(t_{h}, \mathbf{x}_{\mathbf{h}}\right)+\mathbf{R}_{h,+}^{p}$.

2. Otherwise, $t_{h}<T-M \epsilon-h$. By (6.1), for some $(\mathbf{f}, \mathbf{l}) \in \mathrm{FL}^{+}\left(\mathbf{x}_{\mathbf{h}}\right)$, there exist $\widetilde{t}_{h} \in\left(t_{h}+\epsilon+[-2 h, 2 h]\right) \cap \mathbf{R}_{h}$, $\widetilde{\mathbf{x}}_{\mathbf{h}} \in\left(\mathbf{x}_{\mathbf{h}}+\epsilon \mathbf{f}+\alpha_{\epsilon, h} \mathbf{B}\right) \cap \mathbf{R}_{h}^{n}$, and $\widetilde{\mathbf{z}}_{\mathbf{h}} \in\left(\epsilon \mathbf{l}+\alpha_{\epsilon, h} \mathbf{B}\right) \cap \mathbf{R}_{h}^{p}$ such that

$$
\mathbf{z}_{\mathbf{h}} \in \widetilde{\mathbf{z}}_{\mathbf{h}}+V_{\epsilon, h}^{k+1}\left(\widetilde{t}_{h}, \widetilde{\mathbf{x}}_{\mathbf{h}}\right)+\mathbf{R}_{h,+}^{p} \cdot
$$

From the induction assumption, we get:

$$
\mathbf{z}_{\mathbf{h}} \in \widetilde{\mathbf{z}}_{\mathbf{h}}+V_{\epsilon, h}^{k}\left(\widetilde{t}_{h}, \widetilde{\mathbf{x}}_{\mathbf{h}}\right)+\mathbf{R}_{h,+}^{p},
$$

or,

$\mathbf{z}_{\mathbf{h}} \in\left\{\left(\epsilon \mathbf{l}+\alpha_{\epsilon, h} \mathbf{B}\right) \cap \mathbf{R}_{h}^{p}+V_{\epsilon, h}^{k}\left(\left(t_{h}+\epsilon+[-2 h, 2 h]\right) \cap \mathbf{R}_{h},\left(\mathbf{x}_{\mathbf{h}}+\epsilon \mathbf{f}+\alpha_{\epsilon, h} \mathbf{B}\right) \cap \mathbf{R}_{h}^{n}\right):(\mathbf{f}, \mathbf{l}) \in \mathrm{FL}^{+}\left(\mathbf{x}_{\mathbf{h}}\right)\right\}+\mathbf{R}_{h,+}^{p}$.

Applying external stability to

$$
\left.S=\left\{\left(\epsilon \mathbf{l}+\alpha_{\epsilon, h} \mathbf{B}\right) \cap \mathbf{R}_{h}^{p}+V_{\epsilon, h}^{k}\left(t_{h}+\epsilon+[-2 h, 2 h]\right) \cap \mathbf{R}_{h},\left(\mathbf{x}_{\mathbf{h}}+\epsilon \mathbf{f}+\alpha_{\epsilon, h} \mathbf{B}\right) \cap \mathbf{R}_{h}^{n}\right):(\mathbf{f}, \mathbf{l}) \in \mathrm{FL}^{+}\left(\mathbf{x}_{\mathbf{h}}\right)\right\},
$$

and using (6.1) yields $\mathbf{z}_{\mathbf{h}} \in V_{\epsilon, h}^{k+1}\left(t_{h}, \mathbf{x}_{\mathbf{h}}\right)+\mathbf{R}_{h,+}^{p}$.

\section{Proposition 6.3.}

$$
\forall k, \operatorname{Graph}\left(V_{\epsilon, h}^{k}+\mathbf{R}_{h,+}^{p}\right)=A^{k} .
$$

Proof. This relation holds for $k=0$ by definition. Assume now that it holds up to $k$. We aim to prove that it holds for $k+1$, i.e.,

$$
\operatorname{Graph}\left(V_{\epsilon, h}^{k+1}+\mathbf{R}_{h,+}^{p}\right)=A^{k+1} .
$$

First, we prove the inclusion

$$
\operatorname{Graph}\left(V_{\epsilon, h}^{k+1}+\mathbf{R}_{h,+}^{p}\right) \subset A^{k+1} .
$$

Take $\left(t_{h}, \mathbf{x}_{\mathbf{h}}\right) \in I_{h} \times \mathbf{R}_{h}^{n}$ and $\mathbf{z}_{\mathbf{h}} \in V_{\epsilon, h}^{k+1}\left(t_{h}, \mathbf{x}_{\mathbf{h}}\right)+\mathbf{R}_{h,+}^{p}$. Two cases arise:

1. If $t_{h} \geq T-M \epsilon-h$, then by (6.2), we have $\left(t_{h}, \mathbf{x}_{\mathbf{h}}, \mathbf{z}_{\mathbf{h}}\right) \in \operatorname{Graph}\left(V_{\epsilon, h}^{k}+\mathbf{R}_{h,+}^{p}\right)$. Therefore, from the induction assumption, $\left(t_{h}, \mathbf{x}_{\mathbf{h}}, \mathbf{z}_{\mathbf{h}}\right) \in A^{k}$. Moreover, by (5.3), we also have $\left(t_{h}, \mathbf{x}_{\mathbf{h}}, \mathbf{z}_{\mathbf{h}}\right) \in \Gamma_{\epsilon, h}\left(t_{h}, \mathbf{x}_{\mathbf{h}}, \mathbf{z}_{\mathbf{h}}\right)$. Hence, $\left(t_{h}, \mathbf{x}_{\mathbf{h}}, \mathbf{z}_{\mathbf{h}}\right) \in \Gamma_{\epsilon, h}\left(t_{h}, \mathbf{x}_{\mathbf{h}}, \mathbf{z}_{\mathbf{h}}\right) \cap A^{k}$, or $\Gamma_{\epsilon, h}\left(t_{h}, \mathbf{x}_{\mathbf{h}}, \mathbf{z}_{\mathbf{h}}\right) \cap A^{k} \neq \emptyset$, which shows that $\left(t_{h}, \mathbf{x}_{\mathbf{h}}, \mathbf{z}_{\mathbf{h}}\right) \in A^{k+1}$.

2. Otherwise, $t_{h}<T-M \epsilon-h$. By (6.1), for some (f, $\left.\mathbf{l}\right) \in \mathrm{FL}^{+}\left(\mathbf{x}_{\mathbf{h}}\right)$, there exist $\widetilde{t}_{h} \in\left(t_{h}+\epsilon+[-2 h, 2 h]\right) \cap \mathbf{R}_{h}$, $\widetilde{\mathbf{x}}_{\mathbf{h}} \in\left(\mathbf{x}_{\mathbf{h}}+\epsilon \mathbf{f}+\alpha_{\epsilon, h} \mathbf{B}\right) \cap \mathbf{R}_{h}^{n}$, and $\widetilde{\mathbf{z}}_{\mathbf{h}} \in\left(\epsilon \mathbf{l}+\alpha_{\epsilon, h} \mathbf{B}\right) \cap \mathbf{R}_{h}^{p}$ such that

$$
\mathbf{z}_{\mathbf{h}} \in \widetilde{\mathbf{z}}_{\mathbf{h}}+V_{\epsilon, h}^{k}\left(\widetilde{t}_{h}, \widetilde{\mathbf{x}}_{\mathbf{h}}\right)+\mathbf{R}_{h,+}^{p} .
$$

Hence, from the induction assumption, $\left(\widetilde{t}_{h}, \widetilde{\mathbf{x}}_{\mathbf{h}}, \mathbf{z}_{\mathbf{h}}-\widetilde{\mathbf{z}}_{\mathbf{h}}\right) \in A^{k}$. To get that $\left(t_{h}, \mathbf{x}_{\mathbf{h}}, \mathbf{z}_{\mathbf{h}}\right) \in A^{k+1}$, it remains to prove that $\left(t_{h}, \mathbf{x}_{\mathbf{h}}, \mathbf{z}_{\mathbf{h}}\right) \in A^{k}$ and $\left(\widetilde{t}_{h}, \widetilde{\mathbf{x}}_{\mathbf{h}}, \mathbf{z}_{\mathbf{h}}-\widetilde{\mathbf{z}}_{\mathbf{h}}\right) \in \Gamma_{\epsilon, h}\left(t_{h}, \mathbf{x}_{\mathbf{h}}, \mathbf{z}_{\mathbf{h}}\right)$ where $\Gamma_{\epsilon, h}\left(t_{h}, \mathbf{x}_{\mathbf{h}}, \mathbf{z}_{\mathbf{h}}\right)$ is given by $(5.2)$. $\left(t_{h}, \mathbf{x}_{\mathbf{h}}, \mathbf{z}_{\mathbf{h}}\right) \in A^{k}$ comes from Proposition 6.2 and the induction assumption, i.e.,

$$
\left(t_{h}, \mathbf{x}_{\mathbf{h}}, \mathbf{z}_{\mathbf{h}}\right) \in \operatorname{Graph}\left(V_{\epsilon, h}^{k+1}+\mathbf{R}_{h,+}^{p}\right) \subset \operatorname{Graph}\left(V_{\epsilon, h}^{k}+\mathbf{R}_{h,+}^{p}\right)=A_{k} .
$$


Moreover, we have:

(a) $\widetilde{t}_{h} \in\left(t_{h}+\epsilon+[-2 h, 2 h]\right) \cap \mathbf{R}_{h}$,

(b) $\widetilde{\mathbf{x}}_{\mathbf{h}} \in\left(\mathbf{x}_{\mathbf{h}}+\epsilon \mathbf{f}+\alpha_{\epsilon, h} \mathbf{B}\right) \cap \mathbf{R}_{h}^{n}$,

(c) and

$$
\begin{array}{cl} 
& \widetilde{\mathbf{z}}_{\mathbf{h}} \quad \in\left(\epsilon \mathbf{l}+\alpha_{\epsilon, h} \mathbf{B}\right) \cap \mathbf{R}_{h}^{p}, \\
\Rightarrow-\widetilde{\mathbf{z}}_{\mathbf{h}} \quad & \in\left(-\epsilon \mathbf{l}+\alpha_{\epsilon, h} \mathbf{B}\right) \cap \mathbf{R}_{h}^{p}, \\
\Rightarrow \mathbf{z}_{\mathbf{h}}-\widetilde{\mathbf{z}}_{\mathbf{h}} \in\left(\mathbf{z}_{\mathbf{h}}-\epsilon \mathbf{l}+\alpha_{\epsilon, h} \mathbf{B}\right) \cap \mathbf{R}_{h}^{p} .
\end{array}
$$

Hence, $\Gamma_{\epsilon, h}\left(t_{h}, \mathbf{x}_{\mathbf{h}}, \mathbf{z}_{\mathbf{h}}\right) \cap A_{k} \neq \emptyset$ and (6.4) is proved.

Conversely, we prove the inclusion

$$
A^{k+1} \subset \operatorname{Graph}\left(V_{\epsilon, h}^{k+1}+\mathbf{R}_{h,+}^{p}\right) .
$$

Take $\left(t_{h}, \mathbf{x}_{\mathbf{h}}, \mathbf{z}_{\mathbf{h}}\right) \in A^{k+1}$. Two cases arise:

1. If $t_{h} \geq T-M \epsilon-h$. By definition of $A^{k+1},\left(t_{h}, \mathbf{x}_{\mathbf{h}}, \mathbf{z}_{\mathbf{h}}\right) \in A^{k}$. Hence, from the induction assumption, we get $\mathbf{z}_{\mathbf{h}} \in V_{\epsilon, h}^{k}\left(t_{h}, \mathbf{x}_{\mathbf{h}}\right)+\mathbf{R}_{h,+}^{p}$, and from $(6.2), \mathbf{z}_{\mathbf{h}} \in V_{\epsilon, h}^{k+1}\left(t_{h}, \mathbf{x}_{\mathbf{h}}\right)+\mathbf{R}_{h,+}^{p}$.

2. Otherwise, $t_{h}<T-M \epsilon-h$. Then, there exist $\left(\widetilde{t}_{h}, \widetilde{\mathbf{x}}_{\mathbf{h}}, \widetilde{\mathbf{z}}_{\mathbf{h}}\right) \in A^{k}$ such that $\left(\widetilde{t}_{h}, \widetilde{\mathbf{x}}_{\mathbf{h}}, \widetilde{\mathbf{z}}_{\mathbf{h}}\right) \in \Gamma_{\epsilon, h}\left(t_{h}, \mathbf{x}_{\mathbf{h}}, \mathbf{z}_{\mathbf{h}}\right)$, or:

(a) $\tilde{t}_{h} \in\left(t_{h}+\epsilon+[-2 h, 2 h]\right) \cap \mathbf{R}_{h}$,

(b) $\widetilde{\mathbf{x}}_{\mathbf{h}} \in\left(\mathbf{x}_{\mathbf{h}}+\epsilon \mathbf{f}+\alpha_{\epsilon, h} \mathbf{B}\right) \cap \mathbf{R}_{h}^{n}$,

(c) $\widetilde{\mathbf{z}}_{\mathbf{h}} \in\left(\mathbf{z}_{\mathbf{h}}-\epsilon \mathbf{l}+\alpha_{\epsilon, h} \mathbf{B}\right) \cap \mathbf{R}_{h}^{p} \Rightarrow \mathbf{z}_{\mathbf{h}} \in \widetilde{\mathbf{z}}_{\mathbf{h}}+\left(\epsilon \mathbf{l}+\alpha_{\epsilon, h} \mathbf{B}\right) \cap \mathbf{R}_{h}^{p}$,

for some $(\mathbf{f}, \mathbf{l}) \in \mathrm{FL}^{-}\left(\mathbf{x}_{\mathbf{h}}\right)$. From the induction assumption, we have $\widetilde{\mathbf{z}}_{\mathbf{h}} \in V_{\epsilon, h}^{k}\left(\widetilde{t}_{h}, \widetilde{\mathbf{x}}_{\mathbf{h}}\right)+\mathbf{R}_{h,+}^{p}$. Hence,

$$
\mathbf{z}_{\mathbf{h}} \in\left(\epsilon \mathbf{l}+\alpha_{\epsilon, h} \mathbf{B}\right) \cap \mathbf{R}_{h}^{p}+V_{\epsilon, h}^{k}\left(\widetilde{t}_{h}, \widetilde{\mathbf{x}}_{\mathbf{h}}\right)+\mathbf{R}_{h,+}^{p} .
$$

Applying external stability to

$$
\left.S=\left\{\left(\epsilon \mathbf{l}+\alpha_{\epsilon, h} \mathbf{B}\right) \cap \mathbf{R}_{h}^{p}+V_{\epsilon, h}^{k}\left(t_{h}+\epsilon+[-2 h, 2 h]\right) \cap \mathbf{R}_{h},\left(\mathbf{x}_{\mathbf{h}}+\epsilon \mathbf{f}+\alpha_{\epsilon, h} \mathbf{B}\right) \cap \mathbf{R}_{h}^{n}\right):(\mathbf{f}, \mathbf{l}) \in \mathrm{FL}^{+}\left(\mathbf{x}_{\mathbf{h}}\right)\right\},
$$

and using (6.1) yields $\mathbf{z}_{\mathbf{h}} \in V_{\epsilon, h}^{k+1}\left(t_{h}, \mathbf{x}_{\mathbf{h}}\right)+\mathbf{R}_{h,+}^{p}$.

Corollary 6.4. The sequence of approximate set-valued return functions $V_{\epsilon, h}^{k}$ is finite.

Proof. This follows from Proposition 6.3 and the fact that the sequence $A_{k}$ is finite ([4], Prop. 2.18).

We let $k(\epsilon, h)$ denote the smallest index associated with the last element of the sequence in Corollary 6.4.

Corollary 6.5. The epigraph of the approximate set-valued return function $V_{\epsilon, h}^{k(\epsilon, h)}$ converges in the sense of Painlevé-Kuratowski to the epigraph of the set-valued return function $V$, i.e.,

$$
\operatorname{Graph}\left(V+\mathbf{R}_{+}^{p}\right)=\lim _{\epsilon \rightarrow 0^{+}, \frac{h}{\epsilon} \rightarrow 0^{+}} \operatorname{Graph}\left(V_{\epsilon, h}^{k(\epsilon, h)}+\mathbf{R}_{h,+}^{p}\right) .
$$

Proof. From ([4], Thm. 2.19), we have

$$
\operatorname{Viab}_{\phi}(\mathcal{H})=\lim _{\epsilon \rightarrow 0^{+}, \frac{h}{\epsilon} \rightarrow 0^{+}} \overrightarrow{\operatorname{Viab}}_{\Gamma_{\epsilon, h}}\left(\mathcal{H}_{h}\right),
$$

where, in general, $\overrightarrow{V i a b}_{F}(K)$ denotes the discrete viability kernel of a nonempty closed set $K \subset \mathbf{R}^{p}$ for the set-valued map $F$ defined from $\mathbf{R}^{p}$ to $\mathbf{R}^{p}$ ([4], Prop. 2.12). Moreover, from Proposition 4.1, we have

$$
\operatorname{Graph}\left(V+\mathbf{R}_{+}^{p}\right)=\operatorname{Viab}_{\phi}(\mathcal{H}) .
$$

Finally, from Proposition 6.3 and ([4], Prop. 2.18), we have

$$
\operatorname{Graph}\left(V_{\epsilon, h}^{k(\epsilon, h)}+\mathbf{R}_{h,+}^{p}\right)=A^{k(\epsilon, h)}=\overrightarrow{\operatorname{Viab}}_{\Gamma_{\epsilon, h}}\left(\mathcal{H}_{h}\right) .
$$

The desired result follows. 


\section{A general NumERICAL ALGORITHM}

In this section, we present a general algorithm to determine the approximate set-valued return function

$V_{\epsilon, h}^{k(\epsilon, h)}$. As shown in Proposition 7.1, to find $V_{\epsilon, h}^{k(\epsilon, h)}$, it is not necessary to compute $V_{\epsilon, h}^{k}, k=0, \ldots, k(\epsilon, h)$ over their entire domain $I_{h} \times \mathbf{R}_{h}^{n}$.

Proposition 7.1. $\forall k \geq 0, \forall t_{h} \in I_{h}, t_{h} \geq T-M \epsilon-h-k(\epsilon-2 h), \forall \mathbf{x}_{\mathbf{h}} \in \mathbf{R}_{h}^{n}$,

$$
V_{\epsilon, h}^{k+1}\left(t_{h}, \mathbf{x}_{\mathbf{h}}\right)=V_{\epsilon, h}^{k}\left(t_{h}, \mathbf{x}_{\mathbf{h}}\right) .
$$

Proof. For $k=0,(7.1)$ follows directly from (6.2). Assume now that (7.1) holds for some fixed $k>0$. We prove that (7.1) also holds for $k+1$. Let $t_{h} \in I_{h}, t_{h} \geq T-M \epsilon-h-(k+1)(\epsilon-2 h)$ and $\widetilde{t}_{h} \in\left(t_{h}+\epsilon+[-2 h, 2 h]\right) \cap \mathbf{R}_{h}$. Then,

$$
\widetilde{t}_{h} \geq t_{h}+(\epsilon-2 h) \geq T-M \epsilon-h-(k+1)(\epsilon-2 h)+(\epsilon-2 h)=T-M \epsilon-h-k(\epsilon-2 h) .
$$

From the induction assumption, we get $\forall \mathbf{x}_{\mathbf{h}} \in \mathbf{R}_{h}^{n}, \forall(\mathbf{f}, \mathbf{l}) \in \mathrm{FL}^{+}\left(\mathbf{x}_{\mathbf{h}}\right), \forall \widetilde{\mathbf{x}}_{\mathbf{h}} \in\left(\mathbf{x}_{\mathbf{h}}+\epsilon \mathbf{f}+\alpha_{\epsilon, h} B\right) \cap \mathbf{R}_{h}^{n}$,

$$
V_{\epsilon, h}^{k+1}\left(\widetilde{t}_{h}, \widetilde{\mathbf{x}}_{\mathbf{h}}\right)=V_{\epsilon, h}^{k}\left(\widetilde{t}_{h}, \widetilde{\mathbf{x}}_{\mathbf{h}}\right) \text {. }
$$

Hence, using (6.1), we obtain $V_{\epsilon, h}^{k+2}\left(t_{h}, \mathbf{x}_{\mathbf{h}}\right)=V_{\epsilon, h}^{k+1}\left(t_{h}, \mathbf{x}_{\mathbf{h}}\right)$, which completes the proof.

We now present a very general numerical algorithm to approximate the set $V\left(0, \mathbf{x}_{\mathbf{0}}\right)$, where for simplicity, we take the initial state $\mathbf{x}_{\mathbf{0}}$ in $\mathbf{R}_{h}^{n}$. From Corollary 6.5 , the suggested approximation to $V\left(0, \mathbf{x}_{\mathbf{0}}\right)$ is given by the finite set $V_{\epsilon, h}^{k(\epsilon, h)}\left(-h, \mathbf{x}_{\mathbf{0}}\right)$. The proposed numerical algorithm is composed of two stages. In the first stage (Algorithm 7.2), the computational domain is determined using the bound on the dynamics. In the second stage (Algorithm 7.3), $V_{\epsilon, h}^{k(\epsilon, h)}\left(-h, \mathbf{x}_{\mathbf{0}}\right)$ is calculated using the multiple dynamic programming equation (6.1)-(6.2) together with Proposition 7.1.

Choose for example $\epsilon_{i}=1 / 2^{i}$ and $h_{i}=1 / 2^{2 i}$. Let $J$ be the number of discretization steps in $h_{i}$ for the interval $\left[-h_{i}, T+h_{i}\right]$, i.e., $J=\left(T+2 h_{i}\right) / h_{i}+1$ (for simplicity, we assume that $T$ is a multiple of $h_{i}$ ) and let $t_{j}=-h_{i}+j h_{i}$. First, we need to determine the computational domains $\Omega_{j}, j=0, \ldots, J-1$.

Algorithm 7.2. Determination of the computational domain.

Initialization: $\forall t_{j},-h_{i} \leq t_{j}<\epsilon_{i}-3 h_{i}, \Omega_{j}=\left\{\mathbf{x}_{\mathbf{0}}\right\}$. Otherwise, $\Omega_{j}=\emptyset$.

Main loop: $\quad$ Set $j=0$.

1: Repeat

1.1: Set $\mathbf{x}_{\mathbf{h}}$ to the first grid point in $\Omega_{j}$.

1.2: Repeat

1.3: For all $t_{j^{\prime}}, t_{j}+\epsilon_{i}-2 h_{i} \leq t_{j^{\prime}} \leq t_{j}+\epsilon_{i}+2 h_{i}$,

$$
\Omega_{j^{\prime}}=\Omega_{j^{\prime}} \cup\left\{\left(\mathbf{x}_{\mathbf{h}}+\epsilon_{i} \mathbf{f}+\alpha_{\epsilon_{i}, h_{i}} \mathbf{B}\right) \cap \mathbf{R}_{h_{i}}^{n}:(\mathbf{f}, \mathbf{l}) \in \mathrm{FL}^{+}\left(\mathbf{x}_{\mathbf{h}}\right)\right\} .
$$

1.4: Until all the grid points in $\Omega_{j}$ have been visited.

2: Until $j=J-1$.

Algorithm 7.3. Resolution of the multiobjective dynamic programming equation (6.1)-(6.2).

Initialization: $\forall t_{j}, T-M \epsilon_{i}-h_{i} \leq t_{j} \leq T+h_{i}, \forall \mathbf{x}_{\mathbf{h}} \in \Omega_{j}, V_{\epsilon_{i}, h_{i}}^{k\left(\epsilon_{i}, h_{i}\right)}\left(t_{j}, \mathbf{x}_{\mathbf{h}}\right)=\{\mathbf{0}\}$. Otherwise, $V_{\epsilon_{i}, h_{i}}^{k\left(\epsilon_{i}, h_{i}\right)}\left(t_{j}, \mathbf{x}_{\mathbf{h}}\right)=\emptyset$.

Let $j^{*}$ be the largest index such that $t_{j^{*}}<T-M \epsilon_{i}-h_{i}$.

Main loop: Set $j=j^{*}$.

1: Repeat 
1.1: Set $\mathrm{x}_{\mathbf{h}}$ to the first grid point in $\Omega_{j}$ and $A=\emptyset$.

1.2: Repeat

1.3: For all $t_{j^{\prime}}, t_{j}+\epsilon_{i}-2 h_{i} \leq t_{j^{\prime}} \leq t_{j}+\epsilon_{i}+2 h_{i}$,

$$
\left.A=A \cup\left\{\left(\epsilon_{i} \mathbf{l}+\alpha_{\epsilon_{i}, h_{i}} \mathbf{B}\right) \cap \mathbf{R}_{h_{i}}^{p}+V_{\epsilon_{i}, h_{i}}^{k\left(\epsilon_{i}, h_{i}\right)}\left(t_{j^{\prime}},\left(\mathbf{x}_{\mathbf{h}}+\epsilon_{i} \mathbf{f}+\alpha_{\epsilon_{i}, h_{i}} \mathbf{B}\right) \cap \mathbf{R}_{h_{i}}^{n}\right)\right):(\mathbf{f}, \mathbf{l}) \in \mathrm{FL}^{+}\left(\mathbf{x}_{\mathbf{h}}\right)\right\} .
$$

1.4: $\operatorname{Set} V_{\epsilon_{i}, h_{i}}^{k\left(\epsilon_{i}, h_{i}\right)}\left(t_{j}, \mathbf{x}_{\mathbf{h}}\right)=\mathcal{E}(A)$.

1.5: Until all the grid points in $\Omega_{j}$ have been visited.

2: Until $j=0$.

To reduce the size of the set $A$ in Algorithm 7.3, it is possible to keep only the Pareto optimal elements at each iteration in Step 1.3. Using Proposition 2.8, we can change Step 1.3 in Algorithm 7.3 to Step 1.3' as follows:

1.1: Set $\mathrm{x}_{\mathbf{h}}$ to the first point in $\Omega_{j}$ and $A=\emptyset$.

1.2: Repeat

1.3': For all $t_{j^{\prime}}, t_{j}+\epsilon_{i}-2 h_{i} \leq t_{j^{\prime}} \leq t_{j}+\epsilon_{i}+2 h_{i}$,

$$
\left.A=\mathcal{E}\left(A \cup\left\{\left(\epsilon_{i} \mathbf{l}+\alpha_{\epsilon_{i}, h_{i}} \mathbf{B}\right) \cap \mathbf{R}_{h_{i}}^{p}+V_{\epsilon_{i}, h_{i}}^{k\left(\epsilon_{i}, h_{i}\right)}\left(t_{j^{\prime}},\left(\mathbf{x}_{\mathbf{h}}+\epsilon_{i} \mathbf{f}+\alpha_{\epsilon_{i}, h_{i}} \mathbf{B}\right) \cap \mathbf{R}_{h_{i}}^{n}\right)\right):(\mathbf{f}, \mathbf{l}) \in \mathrm{FL}^{+}\left(\mathbf{x}_{\mathbf{h}}\right)\right\}\right) .
$$

1.4: Set $V_{\epsilon_{i}, h_{i}}^{k\left(\epsilon_{i}, h_{i}\right)}\left(t_{j}, \mathbf{x}_{\mathbf{h}}\right)=\mathcal{E}(A)$.

1.5: Until all the points in $\Omega_{j}$ have been visited.

\section{Numerical EXAmples}

In this section, the algorithms from Section 7 are applied to a simple family of optimal control problems for

which the set-valued return function $V$ can be obtained analytically. The convergence of $V_{\epsilon_{i}, h_{i}}^{k\left(\epsilon_{i}, h_{i}\right)}\left(-h_{i}, \mathbf{x}_{\mathbf{0}}\right)$ to $V\left(0, \mathbf{x}_{\mathbf{0}}\right)$ is investigated. Recall that incrementing $i$ by 1 means dividing the discrete time step by 2 and the state mesh width by 4 .

\subsection{Description}

We consider an autonomous biobjective $(p=2)$ problem whose one-dimensional $(n=1)$ dynamics are simply

$$
\dot{x}(s)=u(s), s \in[0, T], x(0)=x_{0},
$$

with $U=\{-1,1\}$. The two cost components of a trajectory $x(\cdot)$ over $I$ is given by

$$
\mathbf{J}_{1}\left(0, x_{0}, u(\cdot)\right)=\int_{0}^{T} P(x(s)) u(s) \mathrm{d} s, \mathbf{J}_{2}\left(0, x_{0}, u(\cdot)\right)=\int_{0}^{T} u(s) \mathrm{d} s,
$$

where $P(\cdot)$ is a given polynomial.

The objective space $Y\left(0, x_{0}\right)$ for the problem above can be determined easily. Clearly,

$$
\mathbf{J}_{2}\left(0, x_{0}, u(\cdot)\right)=\int_{0}^{T} u(s) \mathrm{d} s=\int_{\{u=1\}} \mathrm{d} s-\int_{\{u=-1\}} \mathrm{d} s=\alpha T-(1-\alpha) T=(2 \alpha-1) T,
$$

where $\alpha=\frac{1}{T} \int_{\{u=1\}} \mathrm{d} s$, and

$$
\mathbf{J}_{1}\left(0, x_{0}, u(\cdot)\right)=\int_{0}^{T} P(x(s)) u(s) \mathrm{d} s=\int_{0}^{T} P(x(s)) \dot{x}(s) \mathrm{d} s=[Q(x(t))]_{0}^{T},
$$


where $Q(\cdot)$ is an antiderivative of $P(\cdot)$. With

$$
x(T)=x(0)+\int_{0}^{T} u(s) \mathrm{d} s=x_{0}+(2 \alpha-1) T,
$$

and defining $\delta=(2 \alpha-1) T$, we get

$$
\mathbf{J}_{1}\left(0, x_{0}, u(\cdot)\right)=Q\left(x_{0}+\delta\right)-Q\left(x_{0}\right) \text { and } \mathbf{J}_{2}\left(0, x_{0}, u(\cdot)\right)=\delta .
$$

The set $Y\left(0, x_{0}\right)$ can finally be generated parametrically by varying $\delta$ between $-T$ and $T$.

It is possible to derive a systematic procedure that gives a subinterval of $[-T, T]$ such that for all $\delta$ in this interval, the corresponding element $\left(\mathbf{J}_{1}\left(0, x_{0}, u(\cdot)\right), \mathbf{J}_{2}\left(0, x_{0}, u(\cdot)\right)\right)=\left(Q\left(x_{0}+\delta\right)-Q\left(x_{0}\right), \delta\right)$ in the objective space is Pareto optimal. We therefore assume that the Pareto optimal set $V\left(0, x_{0}\right)$ is known.

\subsection{Results}

We consider four different polynomials $P(\cdot)$ and initial conditions $x_{0}$ with $T=0.5$, which yield four problems of the form above. We have chosen these polynomials such that the Pareto optimal sets $V\left(0, x_{0}\right)$ present different characteristics. For each problem and for $i=3, i=4$, and $i=5$, we compute $V_{\epsilon_{i}, h_{i}}^{k\left(\epsilon_{i}, h_{i}\right)}\left(-h_{i}, x_{0}\right)$ using Algorithms 7.2 and 7.3 from Section 7, provide the cardinality $\operatorname{Card}\left(V_{\epsilon_{i}, h_{i}}^{k\left(\epsilon_{i}, h_{i}\right)}\left(-h_{i}, x_{0}\right)\right)$ of the set $V_{\epsilon_{i}, h_{i}}^{k\left(\epsilon_{i}, h_{i}\right)}\left(-h_{i}, x_{0}\right)$, calculate the Hausdorff distance [2], p. $365 d_{\mathrm{H}}\left(V_{\epsilon_{i}, h_{i}}^{k\left(\epsilon_{i}, h_{i}\right)}\left(-h_{i}, x_{0}\right), V\left(0, x_{0}\right)\right)$ between $V_{\epsilon_{i}, h_{i}}^{k\left(\epsilon_{i}, h_{i}\right)}\left(-h_{i}, x_{0}\right)$ and $V\left(0, x_{0}\right)$, and finally generate a "normalized" Hausdorff distance

$$
\bar{d}_{\mathrm{H}}\left(V_{\epsilon_{i}, h_{i}}^{k\left(\epsilon_{i}, h_{i}\right)}\left(-h_{i}, x_{0}\right), V\left(0, x_{0}\right)\right)=\frac{d_{\mathrm{H}}\left(V_{\epsilon_{i}, h_{i}}^{k\left(\epsilon_{i}, h_{i}\right)}\left(-h_{i}, x_{0}\right), V\left(0, x_{0}\right)\right)}{d_{\mathrm{H}}\left(V_{\epsilon_{3}, h_{3}}^{k\left(\epsilon_{3}, h_{3}\right)}\left(-h_{3}, x_{0}\right), V\left(0, x_{0}\right)\right)} .
$$

Our results are summarized in Tables 1, 2, 3, and 4 and also in Figures 1, 2, 3, and 4. Tables 1, 2, 3, and 4 also contain the size of the corresponding problem. The first number corresponds to the total number of grid points, i.e., the cardinality $\operatorname{Card}\left(\Omega_{j}\right)$ of all the sets $\Omega_{j}$. The second number corresponds to the total number of successors for all the grid points, where a successor to a grid point $\mathbf{x}_{\mathbf{h}}$ is defined as a grid point that can be reached from $\mathbf{x}_{\mathbf{h}}$. Using the terminology of Graph Theory, the size of a problem would correspond to the number of nodes and vertices respectively. Figures 1,2,3, and 4 display the objective space, the Pareto optimal set, and the approximate Pareto optimal sets for $i=3, i=4$, and $i=5$ or each problem.

(MOC1) $P(x)=x-1, x_{0}=1$.

The set $Y\left(0, x_{0}\right)+\mathbf{R}_{+}^{2}$ is convex. Hence, it is possible to obtain every element of $V\left(0, x_{0}\right)$ using the weighting method.

(MOC2) $P(x)=-x+1, x_{0}=1.5$.

The set $Y\left(0, x_{0}\right)-\mathbf{R}_{+}^{2}$ is convex. Only the two Pareto optimal elements for $\delta=-T$ and $\delta=T$ can be obtained using the weighting method.

(MOC3) $P(x)=-2 x^{3}-(15 / 4) x^{2}+(2 / 75) x+1 / 5, x_{0}=0$.

The set $Y\left(0, x_{0}\right)+\mathbf{R}_{+}^{2}$ is nonconvex and the set $V\left(0, x_{0}\right)$ is nonconnected. More precisely, $V\left(0, x_{0}\right)$ is the union of two sets.

(MOC4) $P(x)=-(3 / 2) x-1 / 8, x_{0}=0$.

This problem is similar to (MOC3).

Remark 8.1. To reduce the size of the problems, we have proceeded to some simplifications in Algorithms 7.2 and 7.3. First, we have individually computed $\alpha_{\epsilon_{i}, h_{i}}$ for the dynamics $\mathbf{f}(\cdot, \cdot)$ and each component of the running $\operatorname{cost} \mathbf{L}(\cdot, \cdot)$. Second, we have reduced the interval $\left[t_{j}+\epsilon_{i}-2 h_{i}, t_{j}+\epsilon_{i}+2 h_{i}\right]$ to the single time $t_{j}+\epsilon_{i}-2 h_{i}$. Finally, for any given $\mathbf{l}$, we have reduced the set $\left(\epsilon_{i} \mathbf{l}+\alpha_{\epsilon_{i}, h_{i}} \mathbf{B}\right) \cap \mathbf{R}_{h_{i}}^{p}$ to a single element, i.e., the closest lattice element to $\epsilon_{i} \mathbf{l}$, which somehow corresponds to setting $\alpha_{\epsilon_{i}, h_{i}}=0$ for the running cost. Hence, we have set $M$ to $\max \left\{1, M_{\mathrm{f}}\right\}=1$. 
TABLE 1. Results for (MOC1).

\begin{tabular}{|l|c|c|c|}
\cline { 2 - 4 } \multicolumn{1}{c|}{} & $i=3$ & $i=4$ & $i=5$ \\
\hline Size & $(306,5897)$ & $(1630,65093)$ & $(10422,856445)$ \\
\hline $\operatorname{Card}\left(V_{\epsilon_{i}, h_{i}}^{k\left(\epsilon_{i}, h_{i}\right)}\left(-h_{i}, x_{0}\right)\right)$ & 10 & 33 & 130 \\
\hline$d_{\mathrm{H}}\left(V_{\epsilon_{i}, h_{i}}^{k\left(\epsilon_{i}, h_{i}\right)}\left(-h_{i}, x_{0}\right), V\left(0, x_{0}\right)\right)$ & 0.091227 & 0.046550 & 0.022605 \\
\hline $\bar{d}_{\mathrm{H}}\left(V_{\epsilon_{i}, h_{i}, h_{i}}^{k\left(\epsilon_{i}\right)}\left(-h_{i}, x_{0}\right), V\left(0, x_{0}\right)\right)$ & 1 & 0.5103 & 0.2478 \\
\hline
\end{tabular}

TABLE 2. Results for (MOC2).

\begin{tabular}{|l|c|c|c|}
\cline { 2 - 4 } \multicolumn{1}{c|}{} & $i=3$ & $i=4$ & $i=5$ \\
\hline Size & $(306,10961)$ & $(1630,132125)$ & $(10422,1826357)$ \\
\hline $\operatorname{Card}\left(V_{\epsilon_{i}, h_{i}}^{k\left(\epsilon_{i}, h_{i}\right)}\left(-h_{i}, x_{0}\right)\right)$ & 34 & 130 & 514 \\
\hline$d_{\mathrm{H}}\left(V_{\epsilon_{i}, h_{i}}^{k\left(\epsilon_{i}, h_{i}\right)}\left(-h_{i}, x_{0}\right), V\left(0, x_{0}\right)\right)$ & 0.051067 & 0.033192 & 0.016627 \\
\hline $\bar{d}_{\mathrm{H}}\left(V_{\epsilon_{i}, h_{i}}^{k\left(\epsilon_{i}, h_{i}\right)}\left(-h_{i}, x_{0}\right), V\left(0, x_{0}\right)\right)$ & 1 & 0.65 & 0.3256 \\
\hline
\end{tabular}

TABLE 3. Results for (MOC3).

\begin{tabular}{|l|c|c|c|}
\cline { 2 - 4 } \multicolumn{1}{c|}{} & $i=3$ & $i=4$ & $i=5$ \\
\hline Size & $(306,6529)$ & $(1630,66613)$ & $(10422,834285)$ \\
\hline $\operatorname{Card}\left(V_{\epsilon_{i}, h_{i}}^{k\left(\epsilon_{i}, h_{i}\right)}\left(-h_{i}, x_{0}\right)\right)$ & 3 & 21 & 99 \\
\hline$d_{\mathrm{H}}\left(V_{\epsilon_{i}, h_{i}}^{k\left(\epsilon_{i}, h_{i}\right)}\left(-h_{i}, x_{0}\right), V\left(0, x_{0}\right)\right)$ & 0.765685 & 0.054420 & 0.035360 \\
\hline $\bar{d}_{\mathrm{H}}\left(V_{\epsilon_{i}, h_{i}}^{k\left(\epsilon_{i}, h_{i}\right)}\left(-h_{i}, x_{0}\right), V\left(0, x_{0}\right)\right)$ & 1 & 0.0711 & 0.0462 \\
\hline
\end{tabular}

TABle 4. Results for (MOC4).

\begin{tabular}{|l|c|c|c|}
\cline { 2 - 4 } \multicolumn{1}{c|}{} & $i=3$ & $i=4$ & $i=5$ \\
\hline Size & $(306,7553)$ & $(1630,85213)$ & $(10422,1134221)$ \\
\hline $\operatorname{Card}\left(V_{\epsilon_{i}, h_{i}}^{k\left(\epsilon_{i}, h_{i}\right)}\left(-h_{i}, x_{0}\right)\right)$ & 9 & 33 & 129 \\
\hline$\left.d_{\mathrm{H}}\left(V_{\left.\epsilon_{i}, \epsilon_{i}, h_{i}\right)}\right)\left(-h_{i}, x_{0}\right), V\left(0, x_{0}\right)\right)$ & 0.033857 & 0.028646 & 0.014031 \\
\hline $\bar{d}_{\mathrm{H}}\left(V_{\left.\epsilon_{i}, h_{i}, h_{i}\right)}^{k\left(l_{i}\right)}\left(-h_{i}, x_{0}\right), V\left(0, x_{0}\right)\right)$ & 1 & 0.8461 & 0.4144 \\
\hline
\end{tabular}

\subsection{Discussion}

Because the dynamics and the final time $T$ are the same for all four problems, it is to be expected that the total number of grid points remains the same. On the other hand, the running cost $\mathbf{L}_{1}$ is different for the four problems, hence for a given grid point $\mathbf{x}_{\mathbf{h}}$, the sets $\mathrm{FL}^{+}\left(\mathbf{x}_{\mathbf{h}}\right)$ differ, which explains why the number of successors varies between the four problems.

The large value, i.e., 0.765685, in Table 3 for (MOC3) comes from the fact that the approximate Pareto optimal set is not able for $i=3$ to capture the upper part of the true Pareto optimal set. However, as $i$ increases, the approximate Pareto optimal set now captures the upper part of the Pareto optimal set, and as a result, the Hausdorff distance $d_{\mathrm{H}}\left(V_{\epsilon_{i}, h_{i}}^{k\left(\epsilon_{i}, h_{i}\right)}\left(-h_{i}, x_{0}\right), V\left(0, x_{0}\right)\right)$ decreases considerably.

As $i$ increases, as expected, for the four problems, a better approximation of the true Pareto optimal set is obtained. The proposed approach also works well regardless whether the set $Y\left(0, x_{0}\right)+\mathbf{R}_{+}^{p}$ is convex or not. 


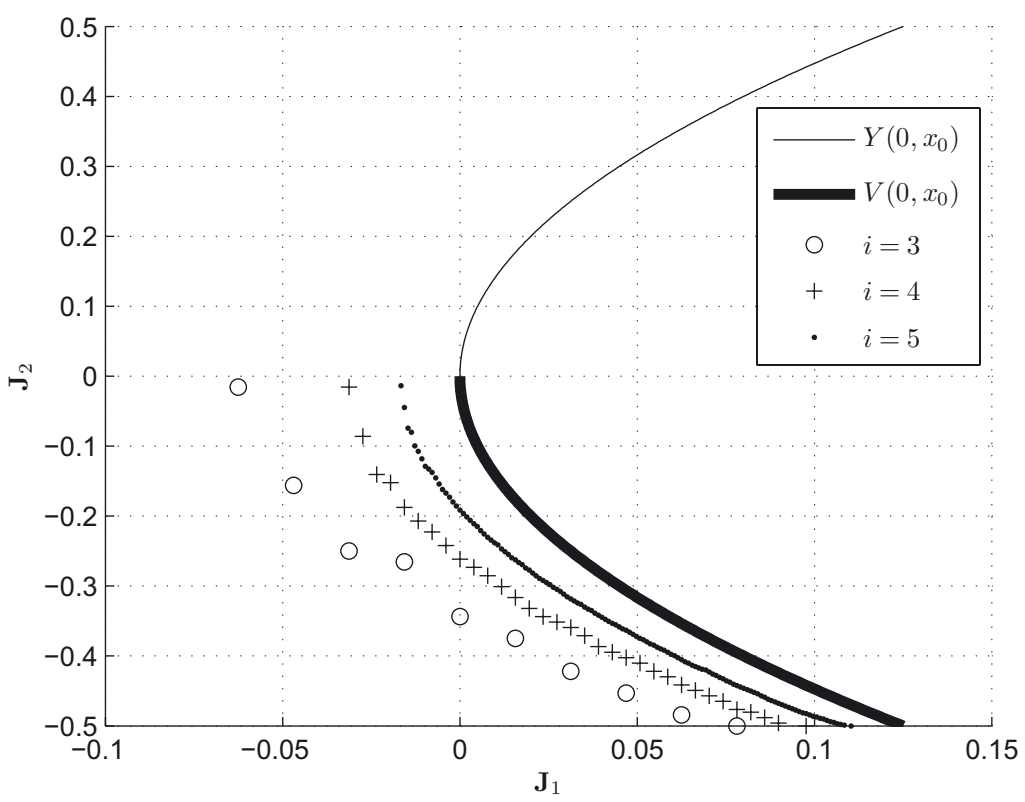

Figure 1. (MOC1): Objective space $Y\left(0, x_{0}\right)$ (plain line), Pareto optimal set $V\left(0, x_{0}\right)$ (bold line) and approximate Pareto optimal set $V_{\epsilon_{i}, h_{i}}^{k\left(\epsilon_{i}, h_{i}\right)}\left(-h_{i}, x_{0}\right)$ with $x_{0}=1$ and for $i=3(\mathrm{o}), i=4$ $(+)$, and $i=5(\cdot)$.

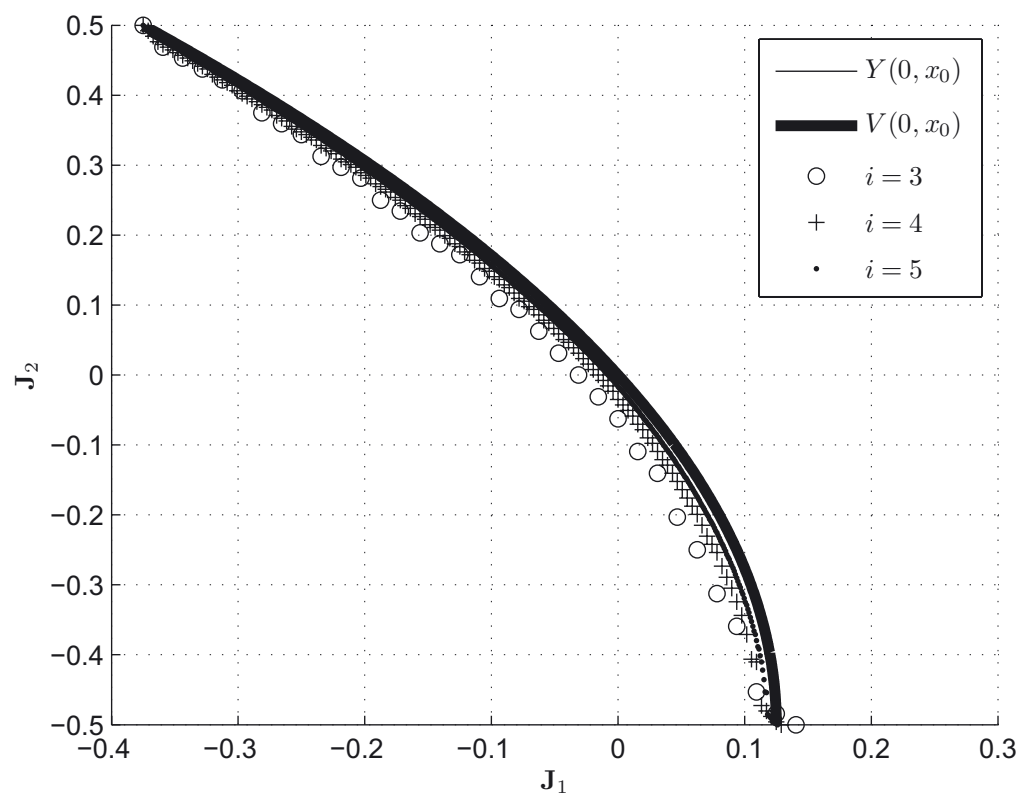

Figure 2. (MOC2): Objective space $Y\left(0, x_{0}\right)$ (plain line), Pareto optimal set $V\left(0, x_{0}\right)$ (bold line) and approximate Pareto optimal set $V_{\epsilon_{i}, h_{i}}^{k\left(\epsilon_{i}, h_{i}\right)}\left(-h_{i}, x_{0}\right)$ with $x_{0}=1.5$ and for $i=3$ (o), $i=4(+)$, and $i=5(\cdot)$. 


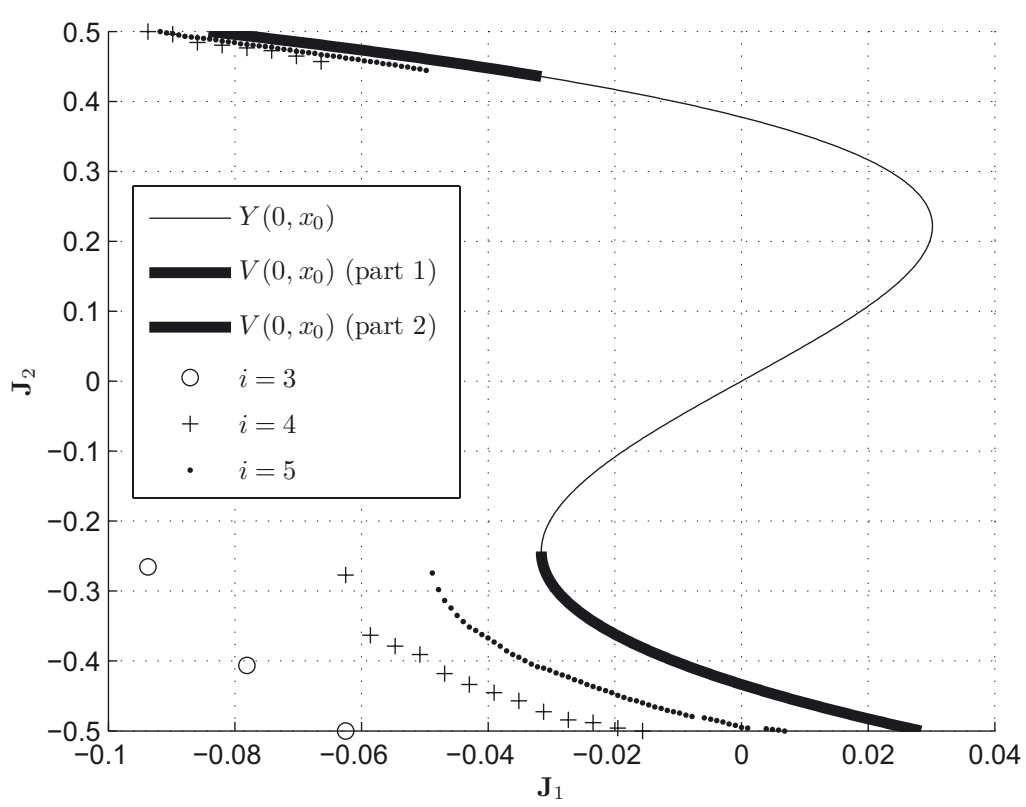

Figure 3. (MOC3): Objective space $Y\left(0, x_{0}\right)$ (plain line), Pareto optimal set $V\left(0, x_{0}\right)$ (bold lines) and approximate Pareto optimal set $V_{\epsilon_{i}, h_{i}}^{k\left(\epsilon_{i}, h_{i}\right)}\left(-h_{i}, x_{0}\right)$ with $x_{0}=0$ and for $i=3$ (o), $i=4(+)$, and $i=5(\cdot)$.

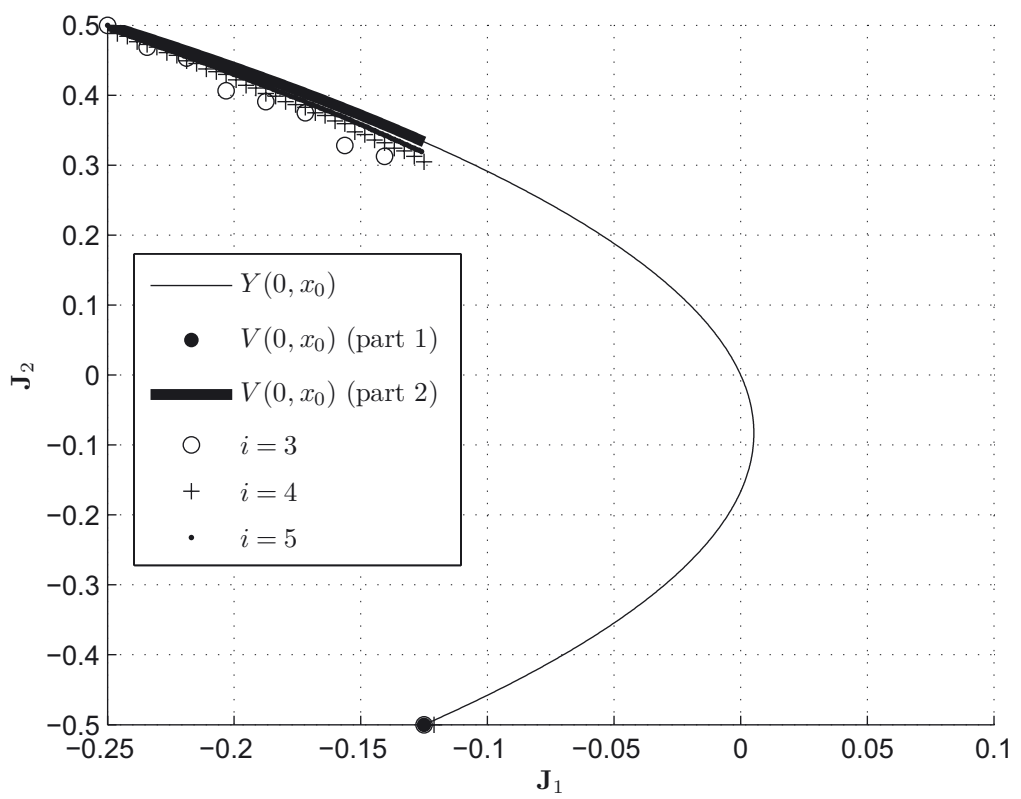

Figure 4. (MOC4): Objective space $Y\left(0, x_{0}\right)$ (plain line), Pareto optimal set $V\left(0, x_{0}\right)$ (bold lines) and approximate Pareto optimal set $V_{\epsilon_{i}, h_{i}}^{k\left(\epsilon_{i}, h_{i}\right)}\left(-h_{i}, x_{0}\right)$ with $x_{0}=0$ and for $i=3$ (o), $i=4(+)$, and $i=5(\cdot)$. 


\section{Conclusion}

In this paper, we have derived a convergent approximation of the Pareto optimal set for finite-horizon multiobjective optimal control problems from the computation of finite discrete viability kernels. A limitation of the proposed approach is the curse of dimensionality induced by the dynamic programming structure underlying this computation. In general, the resolution of high-dimensional problems using dynamic programming remains a challenge and is still an active research topic. We briefly mention domain decomposition [3] or dynamic grid refinement [4] as techniques which have been proposed to address this issue. Closely related to the proposed approach is also the idea of clustering recently presented in [12], which consists of keeping only a subset (with fixed cardinality) of the approximate Pareto optimal set at each grid point during the resolution of the multiobjective dynamic programming equation (6.1)-(6.2).

A direct extension to this work would be to consider the general case of a pointed closed convex cone $P$ instead of the nonnegative orthant $\mathbf{R}_{+}^{p}$ and to allow explicit constraints on the state. Also, the proposed approach could be considered for other classes of optimal control problems, such as multiobjective exit-time optimal control problems [17].

\section{REFERENCES}

[1] J.-P. Aubin, Viability theory. Birkhauser, Boston (1991).

[2] J.-P. Aubin and H. Frankowska, Set-Valued Analysis. Birkhauser, Boston (1990).

[3] M. Bardi and I. Capuzzo-Dolcetta, Optimal Control and Viscosity Solutions of Hamilton-Jacobi-Bellman Equations. Birkhauser, Boston (1997).

[4] P. Cardaliaguet, M. Quincampoix and P. Saint-Pierre, Set-valued numerical analysis for optimal control and differential games. Stochastic and differential games: Theory and numerical methods. Annals of the international Society of Dynamic Games, edited by M. Bardi, T.E.S. Raghavan, T. Parthasarathy. Birkhauser, Boston (1999) 177-247.

[5] P. Cardaliaguet, M. Quincampoix and P. Saint-Pierre, Numerical schemes for discontinuous value functions of optimal control. Set-Valued Anal. 8 (2000) 111-126.

[6] P. Cardaliaguet, M. Quincampoix and P. Saint-Pierre, Differential games through viability theory: Old and recent results. Advances in Dynamic Game Theory. Annals of the international Society of Dynamic Games, edited by S. Jorgensen, M. Quincampoix, T.L. Vincent, T. Basar. Birkhauser, Boston (2007) 3-35.

[7] V. Coverstone-Carroll, J.W. Hartmann and W.J. Mason, Optimal multi-objective low-thrust spacecraft trajectories. Comput. Methods Appl. Mech. Engrg. 186 (2000) 387-402.

[8] A.J. Diaz de Leon and J.C. Seijo, A multi-criteria non-linear optimization model for the control and management of a tropical fishery. Mar. Resour. Econ. 7 (1992) 23-40.

[9] K. Deb, Multi-objective optimization using evolutionary algorithms. John Wiley and Sons, Chichister (2001).

[10] L. Doyen and P. Saint-Pierre, Scale of viability and minimal time of crisis. Set-Valued Anal. 5 (1997) $227-246$.

[11] P.J. Fleming and R.C. Purshouse, Evolutionary algorithms in control systems engineering: a survey. Control Engrg. Pract. 10 (2002) 1223-1241.

[12] A. Guigue, An approximation method for multiobjective optimal control problems application to a robotic trajectory planning problem. Submitted to Optim. Engrg. (2010).

[13] A. Guigue, Set-valued return function and generalized solutions for multiobjective optimal control problems (moc). Submitted to SIAM J. Control Optim. (2011).

[14] A. Guigue, M. Ahmadi, M.J.D. Hayes and R.G. Langlois, A discrete dynamic programming approximation to the multiobjective deterministic finite horizon optimal control problem. SIAM J. Control Optim. 48 (2009) 2581-2599.

[15] A. Guigue, M. Ahmadi, R.G. Langlois and M.J.D. Hayes, Pareto optimality and multiobjective trajectory planning for a 7-dof redundant manipulator. IEEE Trans. Robotics 26 (2010) 1094-1099.

[16] B.-Z. Guo and B. Sun, Numerical solution to the optimal feedback control of continuous casting process. J. Glob. Optim. 39 (1998) 171-195.

[17] A. Kumar and A. Vladimirsky, An efficient method for multiobjective optimal control and optimal control subject to integral constraints. J. Comp. Math. 28 (2010) 517-551.

[18] S. Mardle and S. Pascoe, A review of applications of multiple-criteria decision-making techniques to fisheries. Mar. Resour. Econ. 14 (1998) 41-63.

[19] K.M. Miettinen, Nonlinear Multiobjective Optimization. Kluwer Academic Publishers, Boston (1999).

[20] Y. Sawaragi, H. Nakayama and T. Tanino, Theory of Multiobjective Optimization. Academic Press, Inc., Orlando (1985).

[21] T. Tanino, Sensitivity analysis in multiobjective optimization. J. Optim. Theory Appl. 56 (1988) 479-499.

[22] R. Vinter, Optimal Control. Birkauser, Boston (2000).

[23] P.L. Yu, Cone convexity, cone extreme points, and nondominated solutions in decision problems with multiobjectives. J. Optim. Theory Appl. 14 (1974) 319-377. 